\title{
Zerstörungsfreie Bestimmung elastischer Eigenschaften quadratischer 3-schichtiger Brettsperrholzplatten mit symmetrischem Aufbau
}

\author{
Arne Gülzow • Daniel Gsell • René Steiger
}

Published online: 3 November 2007

(C) Springer-Verlag 2007

Zusammenfassung Die Steifigkeiten von Holzwerkstoffen, wie z.B. von Brettsperrholzplatten, zählen bei statischen Berechnungen zu den wichtigsten Materialeigenschaften. Bei Platten sind es insbesondere die zwei Elastizitätsmoduln $\left(E_{i i}\right)$ in Plattenebene und die drei Schubmoduln $\left(G_{i j}\right)$. Um diese Parameter zerstörungsfrei und mit wenig Arbeitsaufwand bestimmen zu können, wurde ein Verfahren entwickelt, mit dem auf Grundlage von Schwingungsanalysen alle fünf Steifigkeiten in nur einem Versuch bestimmt werden können. Das Verfahren wurde auf 24 aus zwei verschiedenen Herstellerwerken stammenden, quadratischen Brettsperrholzplatten mit Seitenlänge 2,5 m und einer Dicke von $70 \mathrm{~mm}$ mit zwei unterschiedlichen, symmetrischen Aufbauten angewandt. Die so experimentell ermittelten Steifigkeiten wurden mit Biegeversuchen verifiziert und mit auf Grundlage der Verbundtheorie berechneten Steifigkeiten verglichen. Für alle Platten konnten unabhängig von deren Produktionsmethode und Aufbau vier Steifigkeiten sicher bestimmt und die Richtigkeit der Werte verifiziert werden. Die Bestimmbarkeit der Steifigkeit $G_{23}$ ist von der Geometrie der Platte abhängig.

Bemerkenswert ist die Tatsache, dass in der Praxis oft angewendete Steifigkeitsberechnungen, die unter der Annahme durchgeführt wurden, dass das Rohmaterial der einzelnen Schichten der Festigkeitsklasse C24 entspricht, zu optimistische Werte liefern können.

A. Gülzow $(\varpi) \cdot$ D. Gsell · R. Steiger

Empa, Eidgenössische Materialprüfungs- und Forschungsanstalt,

Überlandstrasse 129 ,

8600 Dübendorf, Schweiz

e-mail: arne.guelzow@empa.ch

\begin{abstract}
Non-destructive evaluation of elastic parameters of square-shaped cross-laminated solid wood panels, built up symmetrically with 3 layers
\end{abstract}

\begin{abstract}
Some of the most important material properties of engineered wood products, like cross-laminated solid wood panels, are the elastic properties. Regarding panels, the two in plane MOEs $\left(E_{i i}\right)$ and three shear moduli $\left(G_{i j}\right)$ are of particular interest. In order to determine these parameters with an economically and non-destructive technique, a method was developed which allows to determine all five parameters in one experiment only. This method was approved on 24 square-shaped cross-laminated solid wood panels with side-length of $2.5 \mathrm{~m}$, thickness of $70 \mathrm{~mm}$ and two different layer sizes. The panels were produced by two plants applying different technologies. The determined elastic parameters were verified by bending tests and compared with stiffness parameters calculated on basis of the elastic compound theory. Four elastic parameters could be determined and proven to be correct. The results did neither depend on the different production technologies of the panels nor on the two different compositions of layers. The determinability of the elastic parameter $G_{23}$ depends on the geometry of the panels. Noteworthy is the fact that stiffness parameters calculated assuming that the raw material of the single layers corresponds to strength class C24, can be on the "unsafe side".
\end{abstract}

\section{Einleitung}

Brettsperrholzplattenwerden durch kreuzweise, rechtwinklige Verklebung von aus einzelnen Lamellen zusammengefügten Brettlagen hergestellt und im Ingenieurholzbau als Last abtragende Platten und Scheiben eingesetzt. Die 
Dimensionierung von Holzkonstruktionen hängt überwiegend von Gebrauchstauglichkeitskriterien wie der Beschränkung der maximalen Durchbiegung und Schwingungsamplituden ab. Dies erfordert eine möglichst genaue Kenntnis der elastischen Eigenschaften (Steifigkeiten) des Werkstoffes und der Dämpfungseigenschaften, um Holzkonstruktionen wirtschaftlich, aber auch sicher bemessen zu können.

Zur Bestimmung der elastischen Eigenschaften von Brettsperrholzplatten existieren verschiedene Ansätze:

- Berechnung der Plattensteifigkeiten aus den Eigenschaften der einzelnen Schichten unter Verwendung der Verbundtheorie (Blaß und Görlacher 2003),

- Versuche an Plattenstreifen (EN 789 1995),

- Verfahren nach Gsell et al. (2007), d.h. experimentelle Bestimmung der elastischen Eigenschaften des fertigen Produkts mittels dynamischer Methoden.

Um die elastischen Eigenschaften der Brettsperrholzplatten nach der Verbundtheorie zu berechnen, müssen die mechanischen Eigenschaften der einzelnen Schichten (insb. E-Modul) bekannt sein, was in arbeits- und zeitaufwändigen Experimenten (zerstörungsfreie Prüfung) münden kann.

Die mechanischen Eigenschaften der einzelnen Schichten, bzw. des Rohmaterials, insbesondere die Schub- und Elastizitätsmoduln, können z.B. durch folgende Verfahren bestimmt werden:

- Biegeversuche mit gleich bleibender oder veränderlicher Spannweite (EN 408 2003),

- Messungen der Resonanzfrequenzen eingespannter Balken (Sinclair und Farshad 1987),

- Messungen der Resonanzfrequenzen frei aufliegender Balken (Görlacher 1984, 2002),
- Ultraschall-Laufzeitmessungen (Steiger 1997),

- Verwendung von Sortiermaschinen zur maschinellen Schnittholzsortierung (Boström und Holmqvist 1999).

Für Versuche an Plattenstreifen werden von den Platten parallel zum Plattenrand einzelne Streifen in den Hauptrichtungen abgeschnitten und in 3- oder 4-PunktBiegeversuchen untersucht. Die Platten werden durch das Abschneiden der Streifen zerstört und pro Versuch kann mit dieser Methode nur ein Elastizitäts- oder Schubmodul bestimmt werden, was in einem hohen Zeitaufwand mündet. Des Weiteren werden in solchen Versuchen, wie vorausgegangene Forschungsprojekte zeigten (Czaderski et al. 2007), die elastischen Eigenschaften unterschätzt, da Systemeffekte nicht korrekt erfasst werden.

Gsell et al. (2007) entwickelten ein Verfahren auf Basis der Modalanalyse, mit dem alle für die Biegebeanspruchung maßgebenden elastischen Eigenschaften $\left(C_{11}, C_{22}\right.$, $C_{44}, C_{55}, C_{66}$ (siehe Tabelle 1)) von Brettsperrholzplatten zerstörungsfrei in nur einem Versuch an der gesamten Platte bestimmt werden können. Im vorliegenden Beitrag wird gezeigt, wie diese maßgebenden, globalen Steifigkeiten für quadratische Brettsperrholzplatten mit $2,5 \mathrm{~m}$ Seitenlänge und zwei verschiedenen Aufbauten mit dem Verfahren von Gsell (Gsell et al. 2007) experimentell direkt am fertigen Produkt bestimmt werden können. Um die dynamisch ermittelten Steifigkeiten zu verifizieren, wurden statische Biegeversuche durchgeführt und die gemessenen Durchbiegungen mit berechneten Durchbiegungen unter Einsetzen der dynamisch bestimmten Steifigkeiten verglichen. Ob das unter Herstellern und Holzbauern gebräuchliche Verfahren zur Berechnung der Steifigkeiten (Verbundtheorie und Annahme, sämtliche Schichten entsprechen mindestens der
Tabelle 1 Berechnung der Parameter $C_{i j}$ der Steifigkeitsmatrix Table 1 Calculation of the stiffness matrix parameters $C_{i j}$

\begin{tabular}{lll}
\hline &
\end{tabular}


Festigkeitsklasse C24) zu sicheren und wirtschaftlichen Ergebnissen führt, soll ein Vergleich zwischen berechneten Werten und dynamisch experimentell bestimmten Werten zeigen. Der elastische Parameter $C_{44}\left(G_{23}\right)$ wird zusätzlich gesondert untersucht und dessen Bestimmbarkeit mittels der experimentellen Modalanalyse wird diskutiert.

\section{Material}

Es wurden 24 dreischichtige Platten untersucht. Die Platten wurden von zwei Herstellern gefertigt (Produkt A und Produkt B). Jeder Hersteller produzierte zwölf $70 \mathrm{~mm}$ dicke Platten, neun Platten mit dem Aufbau $10 \mathrm{~mm} / 50 \mathrm{~mm} / 10 \mathrm{~mm}$ und drei Platten mit dem Aufbau $25 \mathrm{~mm} / 20 \mathrm{~mm} / 25 \mathrm{~mm}$ (Abb. 1). Die Platten waren quadratisch mit einer Seitenlänge von 2,5 m. Die Eigenschaften der Einzelschichten der Platten wurden von Czaderski et al. (2007) mittels Ultraschallmessungen vor der Verklebung untersucht.

Produkt A wird aus Schweizer Fichtenholz (Picea abies Karst.) hergestellt. Die Decklagen sind aus Schnittholz gefertigt, das gemäß Herstellerangaben mindestens der Festigkeitsklasse C24 entspricht, in den Mittellagen wird nicht sortiertes Holz eingebaut. Das Besondere an diesen Platten ist deren wirtschaftliche Produktion. Die Decklagen bestehen aus Scheiben, die mit einer Horizontalbandsäge vom liegenden Brettschichtholz abgetrennt werden. Die Mittellagen sind einzelne Fichtenbretter, die von Hand auf den Decklamellen verlegt werden. Die Mittellagenbretter sind seitlich nicht verklebt und weisen einen Abstand von bis zu $5 \mathrm{~mm}$ zur nächsten Lamelle auf. Zur Verklebung wird ein lösungsmittelfreier 1-Komponenten Polyurethankleber verwendet.

Auch Produkt B besteht aus Schweizer Fichtenholz (Picea abies Karst.). Laut Herstellerangaben entspricht es in allen Schichten mindestens der Festigkeitsklasse C24. Die Platten dieses Herstellers werden aus Seitenbrettern gefertigt. Diese werden seitlich zu breiten Lamellen und dann unter Beachtung eines Längsversatzes der Stöße zu einem Blockholz verklebt. Anschließend werden vertikal einzelne beliebig breite Lamellen abgesägt, geschliffen und kreuzweise verklebt. Zur Verklebung wird ein HarnstoffFormaldehyd Klebstoff verwendet. Diese Platten können daher nur in trockenen Innenräumen eingesetzt werden.

\section{Methode}

Um die Steifigkeiten der Brettsperrholzplatten zu bestimmen und diese zu verifizieren, wurde nach dem in Abb. 2 dargestellten Schema vorgegangen. Zuerst wurden die Steifigkeiten aller Platten nach der Verbundtheorie berechnet (erste Zeile Abb. 2). Es wurde angenommen, dass die Herstellerangaben zutreffend sind, d.h. dass sämtliches eingesetztes Rohmaterial der Festigkeitsklasse C24 entspricht und der Ingenieur keine weiteren Informationen über das Material besitzt. Diese Berechnung ist einerseits nötig, um hinreichend genaue Anfangswerte für den später nötigen Optimierungsalgorithmus zu erhalten. Andererseits werden diese berechneten Steifigkeiten auch mit
Abb. 1 Versuchsmaterial: 2 unterschiedliche Produkte und 2 Plattenaufbauten

Fig. 1 Test material: 2 different products and 2 different compositions of layers
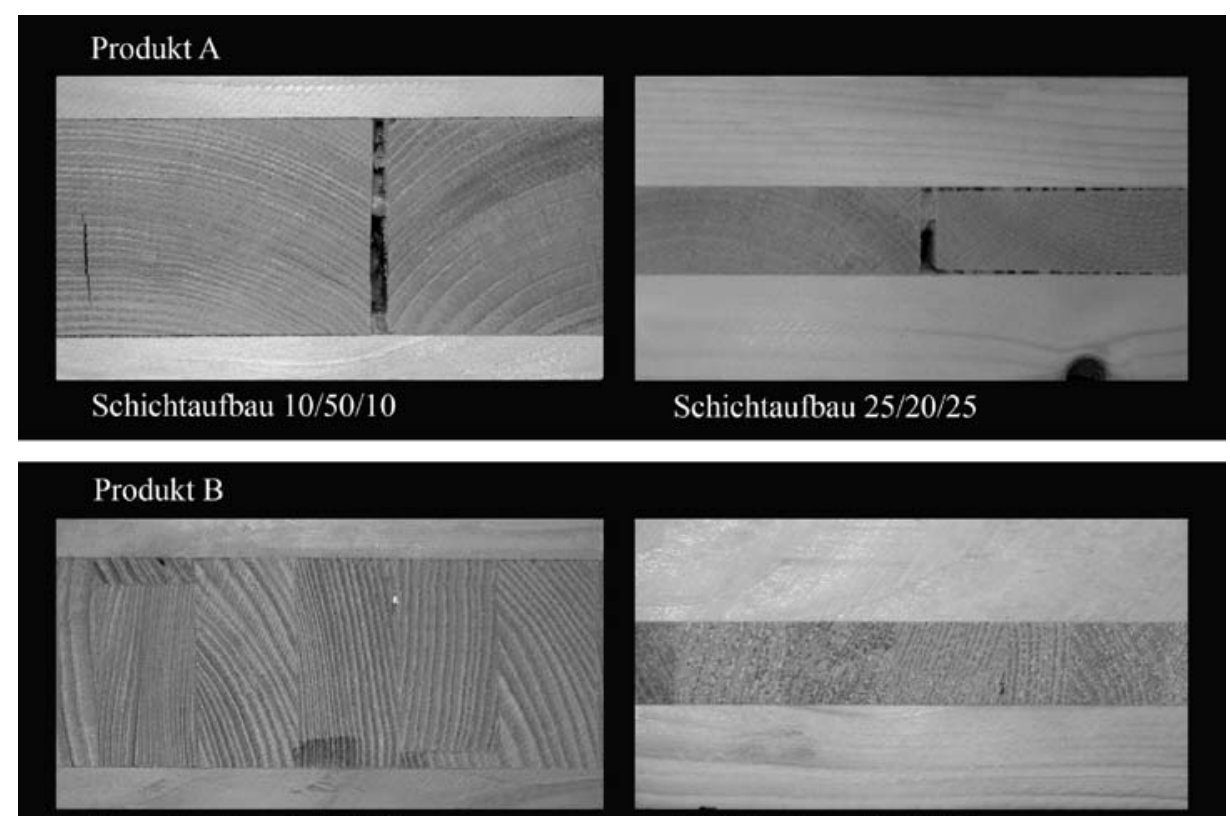

Schichtaufbau 10/50/10

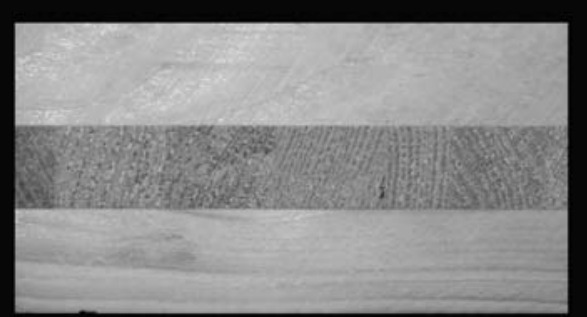

Schichtaufbau 25/20/25 


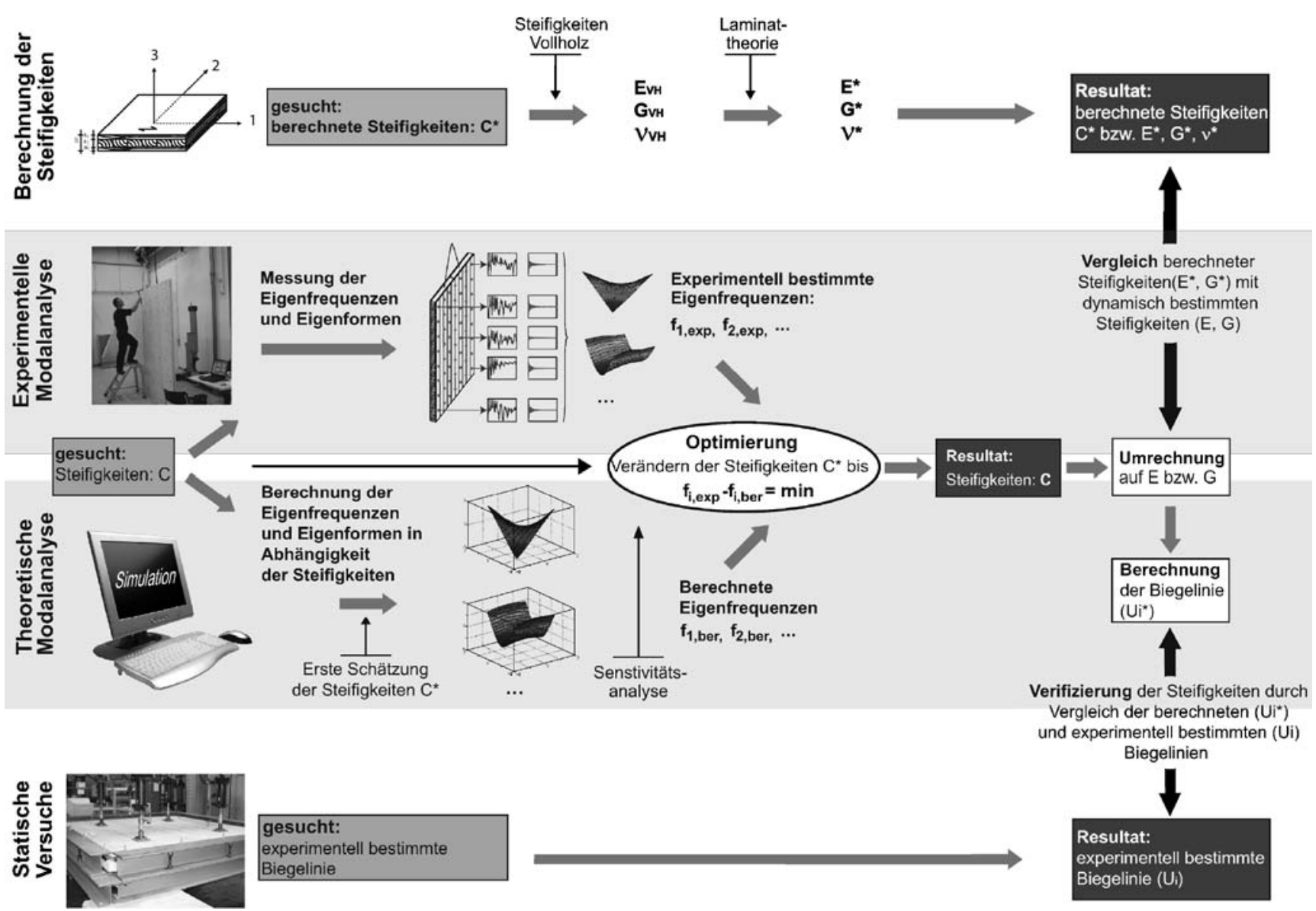

Abb. 2 Schematische Darstellung der angewandten Methode

Fig. 2 Schematic overview of the used method

den dynamisch bestimmten verglichen und diskutiert, ob dieses unter Herstellern und Holzbauern gebräuchliche Verfahren zur Berechnung der Steifigkeiten (Annahme: C24) zu sicheren und wirtschaftlichen Ergebnissen führt. Alle Steifigkeiten werden letztlich auf die im Ingenieurwesen gebräuchlichen Elastizitäts- und Schubmoduln $\left(E_{i i}^{*}\right.$ und $\left.G_{i j}^{*}\right)$ rückgerechnet.

Im Folgenden werden alle berechneten elastischen $\mathrm{Pa}$ rameter mit einem Stern $\left(^{*}\right)$ versehen, um diese klar von den experimentell dynamisch bestimmten elastischen Eigenschaften und Moduln unterscheiden zu können.

Die zweite und dritte Zeile der Abb. 2 erklären das Vorgehen zur zerstörungsfreien Bestimmung der Steifigkeiten mit einer Modalanalyse. Es wurden für alle 24 Platten mit dem Verfahren von Gsell et al. (2007) die Steifigkeiten dynamisch in 3 Schritten bestimmt:

- Experimentelle Modalanalyse,

- Theoretische Modalanalyse,

- Optimierungsprozess.

Anschließend wurden zur Verifikation statische Biegeversuche durchgeführt (unterste Zeile in Abb. 2).

\subsection{Berechnung der elastischen Eigenschaften}

\section{Grundlagen}

Zuerst wurden die elastischen Eigenschaften der Platten mit der Verbundtheorie (Bodig und Jayne 1993) berechnet.

Eine Besonderheit von Holz ist, dass seine Steifigkeiten in Faserrichtung um ein Vielfaches höher sind als senkrecht zur Faserrichtung. Es weist also eine steife und eine weiche Richtung auf, die senkrecht zueinander liegen. Durch die kreuzweise, rechtwinklige Anordnung der einzelnen Lagen entstehen im Brettsperrholz verschiedene Steifigkeiten in die zwei Hauptrichtungen der Platte. Um dem konstruktiven Ingenieur einfache Berechnungsparameter zur Verfügung stellen zu können, werden über die Plattendicke gewichtete, globale orthotrope Eigenschaften angenommen. Das heißt, es existieren drei zueinander senkrechte Achsen, in deren Richtungen die elastischen Eigenschaften unterschiedlich sind (Tabelle 1, Kopf). Die Materialeigenschaften werden über die Plattendicke (sowohl im Mikro- als auch im Makrobereich) als homogen angenommen. Das linear elastische Gesetz für orthotrope Werkstoffe schreibt sich wie 
folgt:

$\sigma_{i j}=C_{i j} \cdot \varepsilon_{i j}$

oder in Matrizenschreibweise:

$\left\{\begin{array}{l}\sigma_{11} \\ \sigma_{22} \\ \sigma_{33} \\ \sigma_{23} \\ \sigma_{31} \\ \sigma_{12}\end{array}\right\}=\left[\begin{array}{cccccc}C_{11} & C_{12} & C_{13} & 0 & 0 & 0 \\ C_{12} & C_{22} & C_{23} & 0 & 0 & 0 \\ C_{13} & C_{23} & C_{33} & 0 & 0 & 0 \\ 0 & 0 & 0 & C_{44} & 0 & 0 \\ 0 & 0 & 0 & 0 & C_{55} & 0 \\ 0 & 0 & 0 & 0 & 0 & C_{66}\end{array}\right] \cdot\left\{\begin{array}{c}\varepsilon_{11} \\ \varepsilon_{22} \\ \varepsilon_{33} \\ 2 \varepsilon_{23} \\ 2 \varepsilon_{31} \\ 2 \varepsilon_{12}\end{array}\right\}$.

Dabei sind $C_{i j}$ die neun unabhängigen Elemente der Steifigkeitsmatrix (elastische Parameter), $\sigma_{i j}$ und $\varepsilon_{i j}$ die Elemente des Spannungs- bzw. Dehnungsvektors (Altenbach et al. 1996). Die neun elastischen Parameter $C_{i j}$ hängen direkt von den Elastizitätsmoduln $E_{i i}$, Schubmoduln $G_{i j}$ und von den Querdehnungszahlen $v_{i j}$ ab. Mit der $C$-Schreibweise kann der später beschriebene Optimierungsalgorithmus einfacher durchgeführt werden. Die $C_{i j}$-Werte sind im Gegensatz zu den Ingenieurskonstanten alle von gleicher Größenordnung und können sehr einfach in die bekannten Ingenieurskonstanten umgerechnet werden (Tabelle 1).

Für statische Berechnungen von Brettsperrholzplatten sind die Parameter $C_{11}, C_{22}, C_{44}, C_{55}, C_{66}$ wichtig. Die Parameter $C_{44}, C_{55}$, und $C_{66}$ entsprechen den Ingenieurskonstanten $G_{23}, G_{13}, G_{12}$. $C_{11}$ und $C_{22}$ sind direkt abhängig von den Ingenieurskonstanten $E_{11}$ und $E_{22}$ und den Querdehnungszahlen $v_{i j}$ (Tabelle 1).
Da bei der Herstellung von Brettsperrholzplatten die Schnittart des Rohmaterials stark unterschiedlich sein kann (Tabelle 2), können die Materialeigenschaften des Vollholzes in den Richtungen 2 und 3 (Tabelle 3), also quer zur Faser, in guter Annäherung als identisch angenommen werden. Des Weiteren wird für die Berechnung, mit Ausnahme der Elastizitätsmoduln $E_{22}$ und $E_{33}$, vorausgesetzt, dass es sich um Holz der Festigkeitsklasse C24 handelt und folgende Abhängigkeiten bestehen:

$E_{11}=11000 \mathrm{~N} / \mathrm{mm}^{2} \quad(\mathrm{EN} 3382003)$,

$G_{13}=G_{12}=E_{1} / 16=688 \mathrm{~N} / \mathrm{mm}^{2} \quad(\mathrm{EN} 3382003)$,

$G_{23}=G_{12} / 10=68,8 \mathrm{~N} / \mathrm{mm}^{2} \quad($ DIN 1052 2004),

$v_{21} / E_{22}=v_{12} / E_{11} ; v_{31} / E_{33}=v_{13} / E_{11} ; v_{23} / E_{22}=v_{32} / E_{33}$,

$E_{22}=E_{33}=E_{11} / 20=550 \mathrm{~N} / \mathrm{mm}^{2} \quad($ Hearmon 1948).

\section{Berechnung der Querdehnungszahlen \\ (Poisson-Konstanten) $v^{*}$}

Um über die Plattendicke gewichtete Querdehnungszahlen von Brettsperrholzplatten zu berechnen, müssen vorerst die Querdehnungszahlen von Vollholz bekannt sein. Tabelle 2 gibt einen Überblick über die in ausgewählten Literaturstellen vorhandenen Angaben zu Querdehnungszahlen. Diese Werte entsprechen jeweils Querdehnungszahlen für radiale, tangentiale und longitudinale Richtungen der Jahrringe. Im Falle der Brettsperrholzplatten, bei welchen

Tabelle 2 Querdehnungszahlen von Holz

Table 2 Poisson ratios of wood

\begin{tabular}{|c|c|c|c|c|c|c|}
\hline Quelle & $v_{L R}$ & $v_{R L}$ & $v_{L T}$ & $v_{T L}$ & $v_{R T}$ & $v_{T R}$ \\
\hline$\overline{\text { DIN } 68364 \text { (1979) }}$ & - & 0,33 & - & - & 0,27 & - \\
\hline (Neuhaus 1994) & 0,06 & 0,41 & 0,04 & 0,55 & 0,31 & 0,60 \\
\hline (Hearmon 1948) & 0,03 & - & - & 0,54 & - & 0,60 \\
\hline$\left(\right.$ Bodig und Jayne 1993) ${ }^{1}$ & 0,04 & 0,37 & 0,03 & 0,42 & 0,35 & 0,47 \\
\hline${\text { (Bodig und Jayne } 1993)^{2}}^{2}$ & 0,03 & 0,40 & 0,02 & 0,52 & 0,30 & 0,51 \\
\hline$\left(\right.$ Albers 1970) ${ }^{2}$ & 0,02 & 0,37 & 0,02 & 0,57 & 0,33 & 0,43 \\
\hline$\left(\right.$ Albers 1970) ${ }^{3}$ & 0,01 & 0,43 & 0,02 & 0,53 & 0,24 & 0,42 \\
\hline$(\text { Stamer } 1935)^{3}$ & 0,02 & 0,43 & 0,01 & 0,53 & 0,24 & 0,42 \\
\hline
\end{tabular}

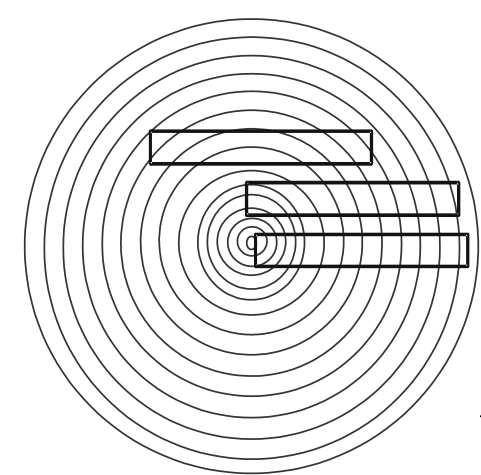

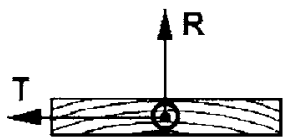

L

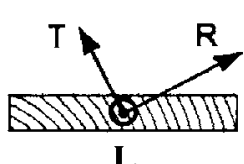

L

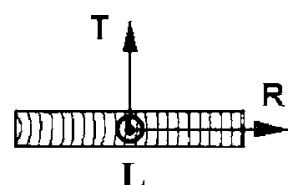

L

\footnotetext{
${ }^{1}$ Softwoods ${ }^{2}$ Spruce ${ }^{3}$ Fichte
} 
Tabelle 3 Berechnung über die Plattendicke gewichteter Querdehnungszahlen, Schubmoduln und Elastizitätsmoduln

Table 3 Calculation of Poisson ratios, shear moduli and moduli of elasticity (MOE), weighted over the thickness of the panels

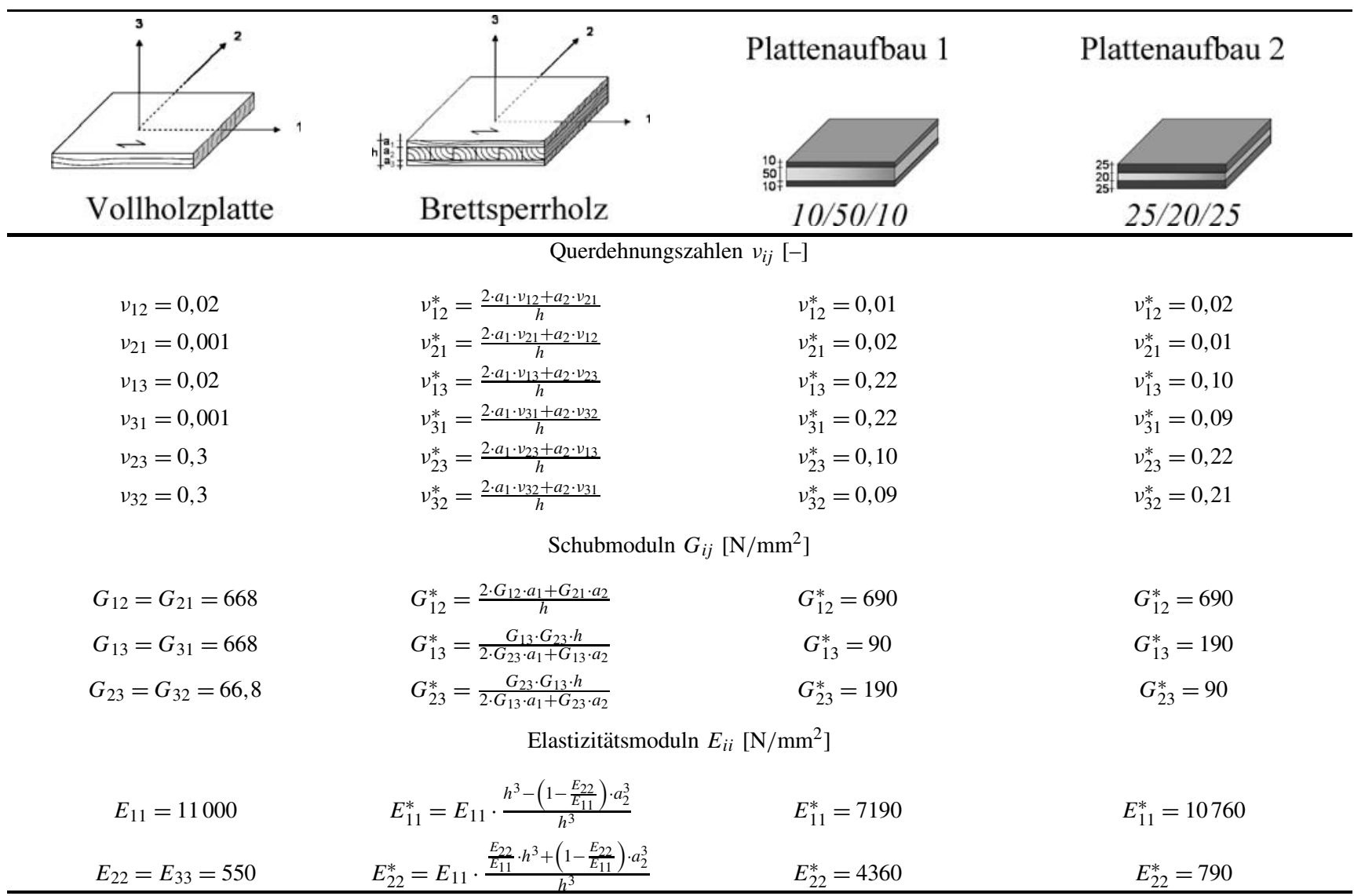

Bretter verschiedener Einschnittarten verwendet werden, ist eine gesonderte Betrachtung der Richtungen ,,Tangential““ und ,Radial“" nicht sinnvoll. Es wird für die Richtungen 2 und 3 des Vollholzes ein Mittelwert aus tangentialer und radialer Richtung angenommen. Somit sind nur zwei verschiedene Querdehnungszahlen nötig. Der dritte Wert wird nach Gl. 5 berechnet. Die für die Berechnungen verwendeten Querdehnungszahlen von Vollholz sind in der ersten Spalte der Tabelle 3 aufgeführt. Des Weiteren sind in Tabelle 3 die Formeln zur Berechnung gewichteter Querdehnungszahlen in Sandwich-Elementen in Anlehnung an Bodig und Jayne (1993) und die berechneten Querdehnungszahlen für die beiden Plattenaufbauten angegeben.

\section{Berechnung der Schubmoduln $G^{*}$}

Die Schubmoduln von Vollholz können nach Gl. 3 und 4 näherungsweise berechnet werden. Aufgrund der Annahme, dass Brettsperrholz über die Plattendicke homogen ist, müssen auch hier gewichtete Schubmoduln berechnet werden. Das geschieht über eine flächenanteilsmäßige Mittelung der Schubmoduln der einzelnen Schichten. In Tabelle 3 sind die Ausgangswerte der Schubmoduln von Voll- holz, Formeln für die Berechnung von Schubmoduln für Sandwich-Elemente und die berechneten Schubmoduln der Brettsperrholzplatten für die beiden Aufbauten angegeben.

\section{Berechnung der Elastizitätsmoduln $E^{*}$}

Berechnet wurden die E-Moduln unter Berücksichtigung der Querlagen und der Annahme, dass die Querschnitte bei Biegung eben bleiben. Die Biegesteifigkeit der Platte setzt sich aus der Summe der Biegesteifigkeiten der einzelnen Lagen und deren Steineranteilen zusammen. Allgemein ist diese Berechnungsmethode ,gewichteter" Steifigkeiten als Verbundtheorie bekannt. Zur Berechnung kann auf zusammengefasste Gleichungen nach Blaß und Görlacher (2003) zurückgegriffen werden. Die Formeln zur Berechnung der ,gewichteten“ E-Moduln für dreischichtige Platten und die berechneten E-Moduln sind in Tabelle 3 angegeben.

\subsection{Experimentelle Modalanalyse}

In der experimentellen Modalanalyse (zweite Zeile in Abb. 2) werden die Eigenfrequenzen $\left(f_{i, \exp }\right)$ und Eigenformen (,Modes“) der Platten im Versuch bestimmt. Da 
die Feuchtigkeit des Holzes einen großen Einfluss auf die elastischen Eigenschaften des Materials hat, wurden die Experimente in einem klimatisierten Raum $\left(20^{\circ} \mathrm{C}, 60 \%\right)$ durchgeführt, was eine mittlere Holzausgleichsfeuchte von $11 \%$ erwarten lässt. Die Feuchtigkeitsmessungen kurz vor den Versuchen ergaben im Mittel Feuchtigkeitsgehalte von 10,2\% in den Decklagen und 12,3\% in den Mittellagen.

In dynamischen Versuchen sind Randbedingungen wie Einspannungen oder einfache Auflager technisch nur schwierig realisierbar, da immer ein Teil der mechanischen Energie abfließt, anstatt vollständig reflektiert zu werden. Um freie Ränder zu realisieren, wurden die Platten deshalb an leichten, dünnen und langen Schnüren aufgehängt. Mit einem Impulshammer, der die Intensität des Schlages misst, wurden die Platten an 36 Punkten angeschlagen (Abb. 3), welche auf einem gleichmäßigen Raster von 6 mal 6 Punkten angeordnet waren. Die Schwingungen wurden mit kleinen, leichten Beschleunigungssensoren gemessen, die auf der Rückseite der Platten (zwei im Bereich der Ecken und einer einige Zentimeter neben der Mitte) angebracht wurden. Eine Analyse im Vorfeld der Messungen ergab, dass sich diese Messstellen am besten eigneten, da sich hier bei fast allen Eigenformen keine Knotenpunkte einstellten. Aus den aufgezeichneten Messwerten wurden die Eigenfrequenzen und die entsprechenden Eigenformen der Platten bestimmt.

\subsection{Theoretische Modalanalyse}

In der theoretischen Modalanalyse (dritte Zeile von Abb. 2) wird exakt der gleiche Versuch wie bei der experimentel- len Modalanalyse im Computer nachgebildet, mit dem Ziel, die Eigenfrequenzen $\left(f_{i, \text { ber }}\right)$ und die Eigenformen der frei schwingenden Platte in Abhängigkeit der elastischen Parameter $C_{i j}$ zu berechnen. Während die Dichte und die Geometrie der Platte bekannt sind, sind die einzigen Unbekannten in dieser Berechnung die gesuchten elastischen Parameter $C_{i j}$ der Platte. Diese werden in einem ersten Rechendurchgang mit den in Abschn. 3.1 berechneten elastischen Eigenschaften $\left(C_{i j}^{*}\right)$ geschätzt.

Um auch Schubdeformationen zu berücksichtigen, wurde auf die Plattentheorie nach Reddy (1984) zurückgegriffen, mit welcher die Verformungen der Platte (Dicke $h=$ $2 \cdot h_{1}$ ) beschrieben werden. Reddy führt im Verschiebungsfeld der Platte Rotationsfreiheitsgrade ein, die neben der linearen Abhängigkeit in Dickenrichtung zusätzlich quadratische und kubische Terme enthalten. Die Randbedingungen $\sigma_{13}\left(z= \pm h_{1}\right)=0$ und $\sigma_{23}\left(z= \pm h_{1}\right)=0$ führen zu folgendem dreidimensionalen Verschiebungsfeld:

$$
\left\{\begin{array}{l}
u_{1} \\
u_{2} \\
u_{3}
\end{array}\right\}=\left[\begin{array}{ccc}
z-\frac{z^{3}}{3 h_{1}^{2}} & 0 & \frac{-z^{3}}{3 h_{1}} \cdot \frac{\partial}{\partial x} \\
0 & z-\frac{z^{3}}{3 h_{1}^{2}} & \frac{-z^{3}}{3 h_{1}} \cdot \frac{\partial}{\partial y} \\
0 & 0 & 1
\end{array}\right] \cdot\left\{\begin{array}{c}
\psi_{1}(x, y) \\
\psi_{2}(x, y) \\
u_{30}(x, y)
\end{array}\right\} .
$$

$u_{30}$ ist die Querverschiebung der Plattenmittelfläche und $\psi_{i}$ sind die Rotationen der Normalen zur Mittelebene. Somit wird angenommen, dass die Verschiebung $u_{3}$ unabhängig von der Dickenkoordinate ist, was im betrachteten Frequenzbereich zulässig ist. In der Zeit wird ein harmonischer Ansatz, mit $\omega$ als Kreisfrequenz, angenommen.
Abb. 3 Hängende Platte mit gleichmäßigem Raster von $6 \times 6$ Punkten

Fig. 3 Hanging panel with Cartesian grid of 6 times 6 equally spaced points
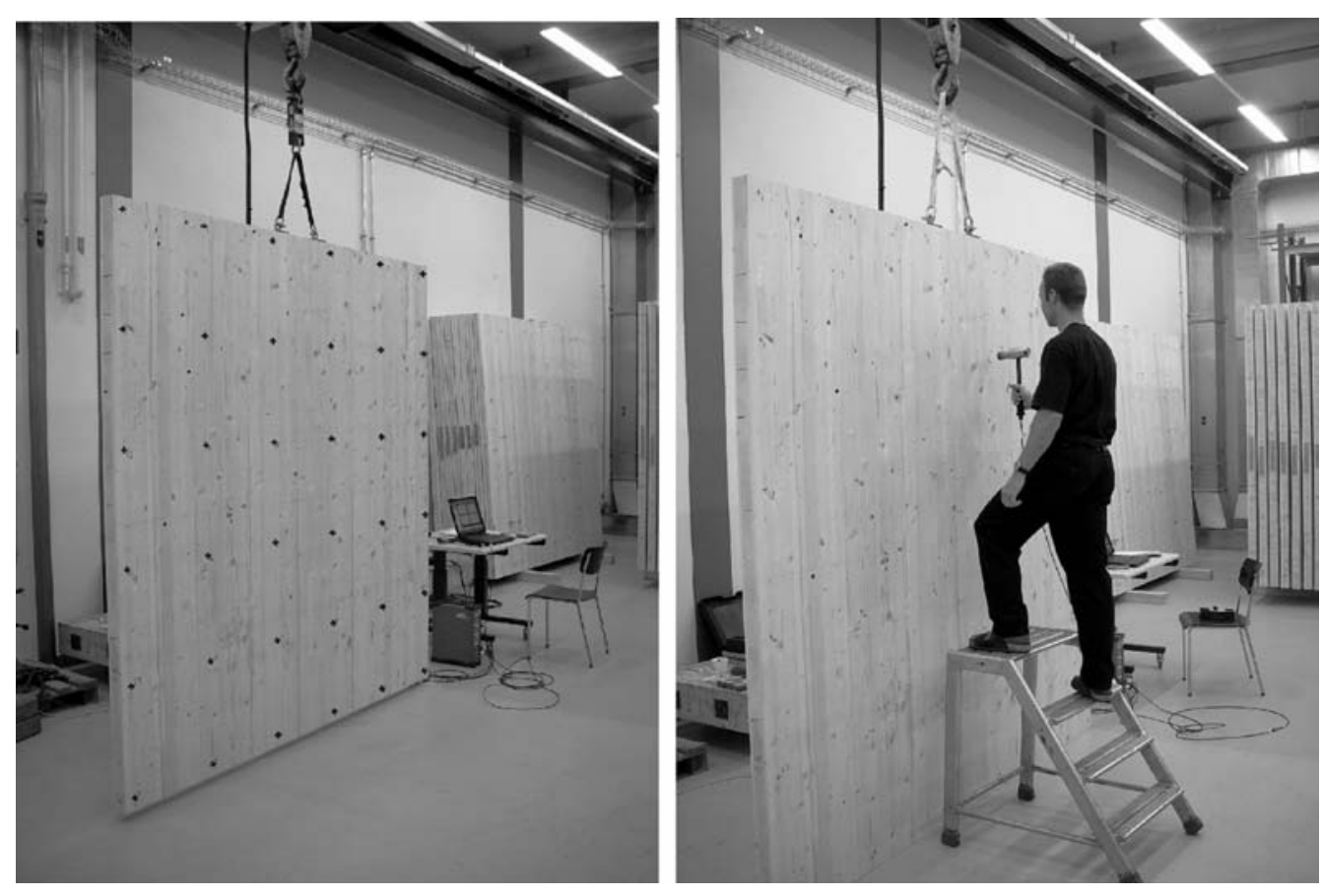
Die Rotations- und Verschiebungsfelder werden mit dem Produkt von zwei vollständigen Reihen aus LegendrePolynomen parametrisiert. Durch Anwendung des Hamilton'schen Prinzips können die Resonanzfrequenzen und die Schwingungsformen bestimmt werden. Hierfür wird die Änderung der Gesamtenergie, die sich aus dem elastischen Potential und der kinetischen Energie zusammensetzt, Null gesetzt, woraus folgendes Eigenwertproblem resultiert:

$(\boldsymbol{K}-\omega \cdot \boldsymbol{M}) \cdot \boldsymbol{q}=\mathbf{0}$

mit $\boldsymbol{K}$ als Steifigkeitsmatrix und $\boldsymbol{M}$ als Massenmatrix.

Einzelheiten zum Verfahren und den angewendeten Modellen können Gsell et al. (2007) entnommen werden.

\subsection{Optimierungsprozess}

In beiden Modalanalysen (experimentelle und theoretische) werden Eigenfrequenzen und Eigenformen ermittelt. Während im Experiment die ,realen“ Frequenzen bestimmt werden $\left(f_{i, \exp }\right)$, handelt es sich bei den berechneten $\left(f_{i, \text { ber }}\right)$ um erste Näherungswerte, welche von den geschätzten Steifigkeiten $\left(C_{i j}^{*}\right)$ abhängen.

Aufgabe des Optimierungsprozesses ist es, die Steifigkeiten im theoretischen Modell so lange systematisch anzugleichen, bis der Unterschied zwischen den theoretisch berechneten und den experimentell bestimmten Eigenfrequenzen minimal wird. Dies geschieht mit Hilfe eines Optimierungsalgorithmus, dessen Resultat diejenigen Steifigkeiten sind, welche das Rechenmodell am besten an das Experiment anpasst. Somit sind die gesuchten Steifigkeiten $C_{i j}$ bestimmt.

Da es sich anfänglich lediglich um grobe Schätzungen handelt, stimmt die Reihenfolge der experimentell bestimmten und der theoretisch berechneten Schwingungsformen nicht zwingend überein. Um sicherzustellen, dass nur die Frequenzen von jeweils zusammengehörenden Schwingungsformen in die Schätzung der elastischen Eigenschaften einfließen, werden MAC (Modal Assurance Criterion) Werte verwendet (Maia 1997). Sie geben an, wie gut die theoretisch und die experimentell bestimmten Eigenformen übereinstimmen.

\section{Sensitivitätsanalyse}

Der Optimierungsalgorithmus konvergiert nur, wenn sich beim Ändern der einzelnen Steifigkeiten auch die berechneten Eigenfrequenzen der Biegeschwingungen genügend stark ändern. Daher wurde eine Sensitivitätsanalyse durchgeführt. Hierbei wurde jeder einzelne Steifigkeitsparameter $C_{i j}$ um $10 \%$ erhöht und der Einfluss dieser Änderungen auf die Resonanzfrequenzen untersucht. Es wurde analog zu Gsell et al. (2007) festgestellt, dass die Resonanzfrequenzen eng von den Steifigkeitsparametern $C_{11}, C_{22}, C_{55}$ und $C_{66}$ abhängig sind und nur wenig von $C_{44}$ beeinflusst werden. Dies hängt damit zusammen, dass die Bestimmbarkeit von $C_{44}$ von der Geometrie der Platte, also der Dicke, den Seitenverhältnissen und dem Schichtaufbau abhängig ist. Die Scheibensteifigkeit $C_{66}\left(G_{12}\right)$ kann aus den Biegeschwingungen abgeleitet werden, da die Frequenzen der so genannten Torsionsmodes (Mode 1, 4, 5, 6, 9, 10, 13 und 19 in Tabelle 6) sehr sensitiv auf eine Änderung von $C_{66}$ reagieren.

\section{Berechnung der Durchbiegung}

Mit Hilfe eines statischen Berechnungsmodells, unter Verwendung der im Optimierungsprozess dynamisch bestimmten Steifigkeitsparameter $C_{i j}$, wurden die Durchbiegungen $\left(u_{i}^{*}\right)$ der Platten an den Stellen, an welchen sie später in den statischen Biegversuchen gemessen wurden (Abschn. 3.5), berechnet und schließlich mit den gemessenen Durchbiegungen $\left(u_{i}\right)$ verglichen.

Das statische Modell basiert auf denselben mechanischen Grundüberlegungen wie das dynamische Modell (siehe Abschn. 3.3). Bei der Formulierung des Hamilton'schen Prinzips entfällt der Anteil der kinetischen Energie, jedoch muss die äußere Arbeit, also die Belastung, berücksichtigt werden. Die Auflagerbedingungen werden erfüllt, indem die parametrisierten Verschiebungsfelder mit Funktionen multipliziert wurden, welche die Randbedingungen der allseitig einfach aufgelegten Platte erfüllen.

\subsection{Statische Biegeversuche}

Die dynamisch bestimmten Steifigkeiten wurden mit Biegeversuchen an allen 24 Platten verifiziert (Czaderski et al. 2007). Die Platten waren allseitig auf ein $50 \mathrm{~mm}$ breites Linienlager aufgelegt und gegen Abheben gesichert. Die Lasteinleitung erfolgte über quadratische Stahlplatten mit Seitenlängen von $150 \mathrm{~mm}$. Die Kraft wurde durch Pendelmanometer mittels Öldruck erzeugt und durch DMSZug-Druck-Kraftaufnehmer gemessen. Die Durchbiegungen wurden mit mechanischen Messuhren an 4 Punkten gemessen. Die Messstellen sind in Abb. 4 dargestellt und befinden sich immer genau im Viertels- oder Zentrumspunkt der Platte $(612,5 \mathrm{~mm}$ oder $1225 \mathrm{~mm}$ vom Plattenrand entfernt). Das Versuchsprogramm beinhaltete Belastungen mit vier Einzellasten in den Quadrantenmitten, einer Einzellast in Plattenmitte sowie in einem der Quadrantenmittelpunkte. Alle Platten wurden stufenweise bis $\mathrm{zu}$ einer maximalen Last von $30 \mathrm{kN}$ belastet und anschließend wieder entlastet. Eine Übersicht über die Belastungsanordnung der einzelnen Platten gibt Tabelle 4.

Um festzustellen, ob die Verifizierung der dynamisch bestimmten Steifigkeiten durch statische Versuche für alle Steifigkeitsparameter aussagekräftig ist und welche Steifig- 
Abb. 4 Berechnete

Verformungsfiguren,

Belastungspunkte und

Messstellen (U1-U4)

Fig. 4 Calculated deformation

shapes, loading points and

measuring points $(U 1-U 4)$
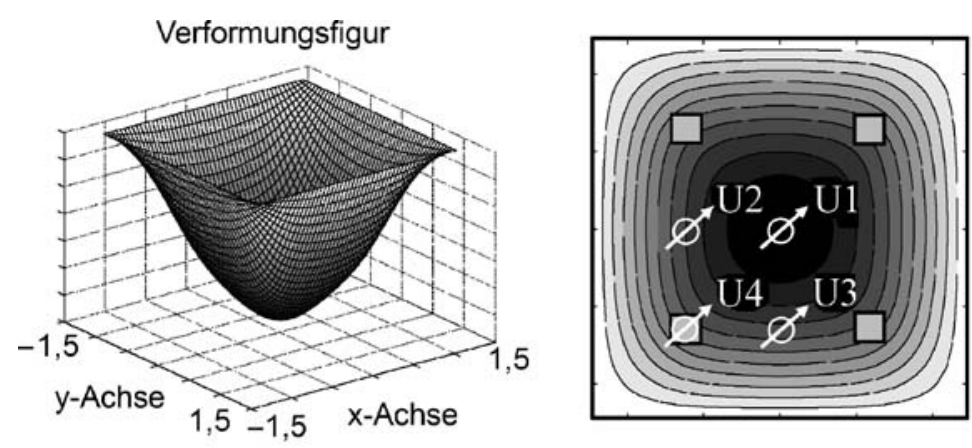

Schichtaufbau

$10 / 50 / 10$

4 Einzellasten in
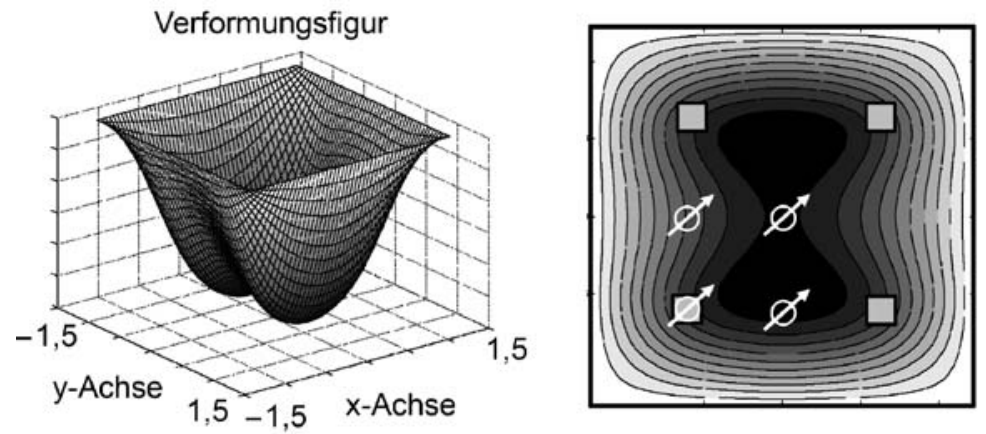

den Quadranten-

mitten

Schichtaufbau

$25 / 20 / 25$

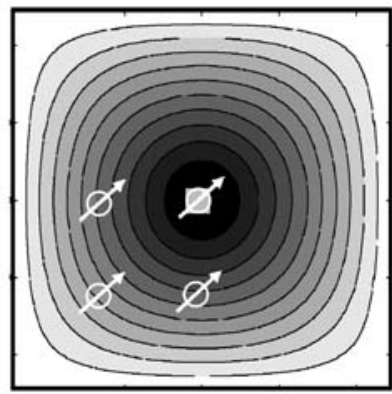

1 zentrische

Einzellast

Schichtaufbau

$10 / 50 / 10$
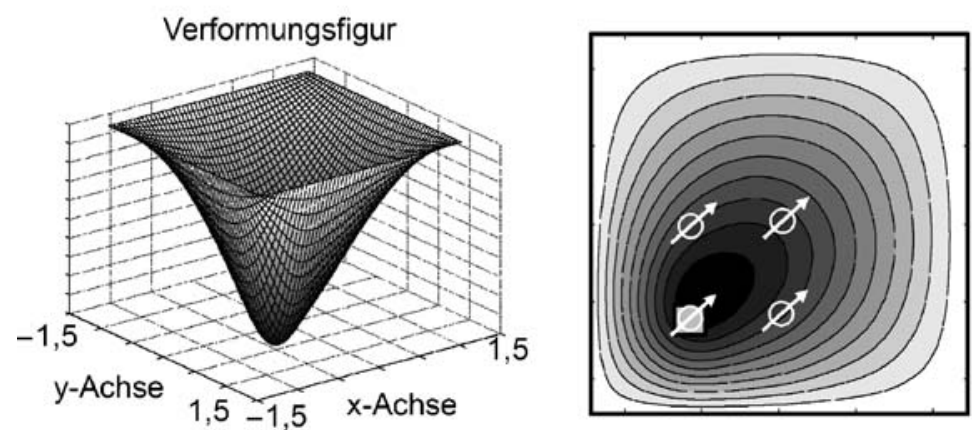

1 exzentrische

Einzellast in

Quadrantenmitte

Schichtaufbau

$10 / 50 / 10$

keitsparameter für die statische Berechnung von Brettsperrholzplatten benötigt werden, wurde eine Parameterstudie durchgeführt. Hierbei wurde für jeden Plattenaufbau und jede Belastungssituation eine repräsentative Platte ausgewählt. Deren experimentell dynamisch bestimmte Steifigkeitsparameter wurden jeweils um $10 \%$ erhöht und der Einfluss jedes Steifigkeitsparameters auf die berechneten Durchbiegungen kontrolliert. In den Diagrammen der Ta- belle 5 sind nur die für die Berechnung der Durchbiegungen wichtigsten Steifigkeitsparameter $C_{11}, C_{22}, C_{44}, C_{55}$ und $C_{66}$ dargestellt. Alle anderen wiesen einen vernachlässigbar kleinen Einfluss auf die Durchbiegung auf. Es ist gut zu erkennen, dass die maximale Änderung des Steifigkeitsparameter $C_{44}$ nur $0,2 \%$ beträgt. Die Genauigkeit dieses Parameters kann somit mit diesen Versuchen nicht verifiziert werden. 
Tabelle 4 Dynamisch experimentell bestimmte Steifigkeiten $C_{i j}, G_{i j}$ und $E_{i i}$ der Platten

Table 4 Experimentally determined dynamic stiffness parameters $C_{i j}, G_{i j}$ and $E_{i i}$ of the panels

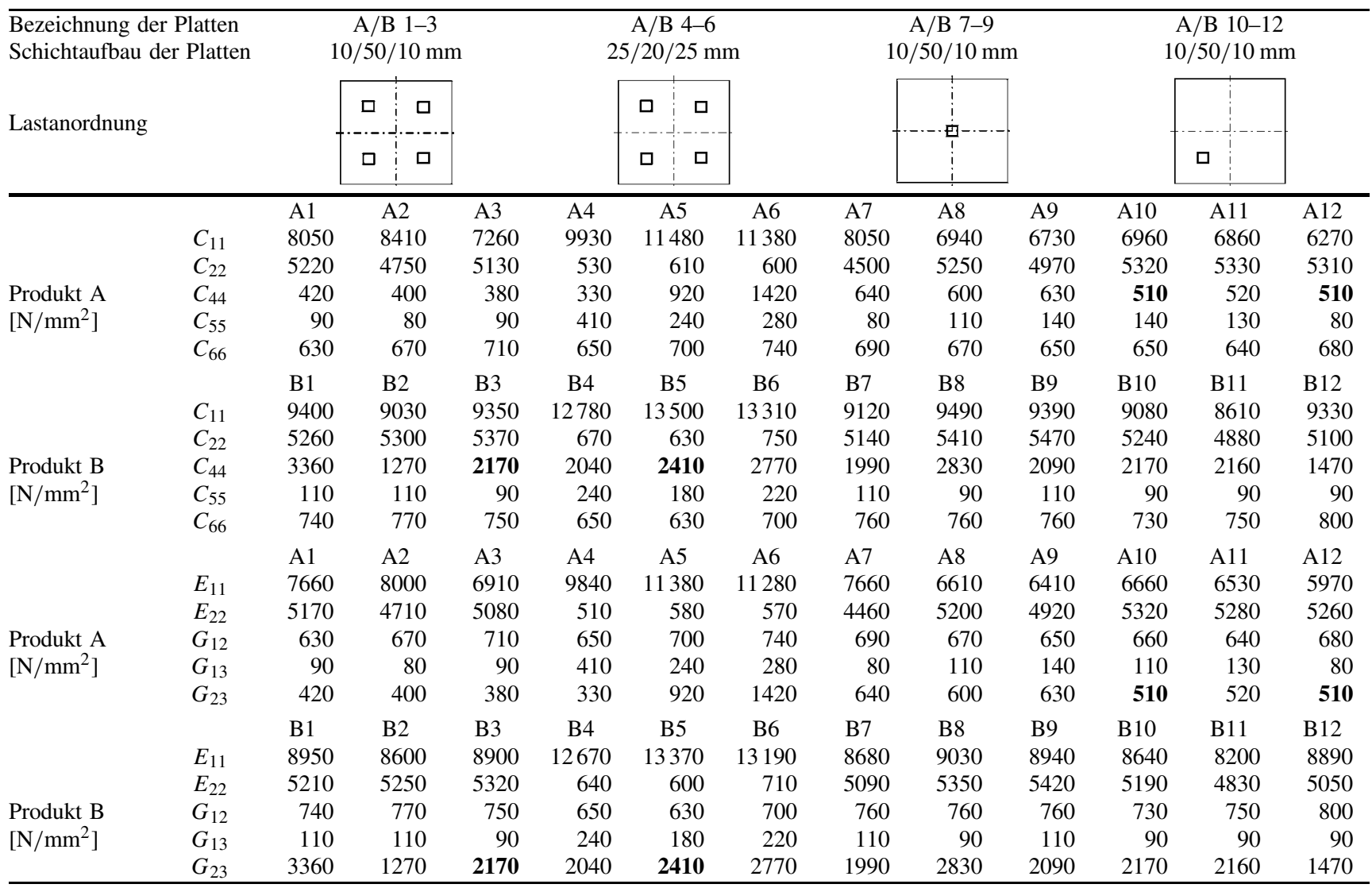

\section{Resultate}

\subsection{Erkenntnisse aus der Anwendung der Methode}

Da es sich bei dem untersuchten Verfahren um eine neue Methode zur Bestimmung der Steifigkeiten von Holzplatten handelt und diese Anwendung das Potenzial besitzt, im Produktionsfluss eingesetzt $\mathrm{zu}$ werden, wurde besonderes Augenmerk auf den Durchführungsprozess gelegt.

\section{Dynamisch bestimmte Steifigkeiten}

Für beinahe alle Platten konnten die Steifigkeiten $C_{11}, C_{22}$, $C_{44}, C_{55}, C_{66}$ bestimmt werden. Ausnahme bildeten die Steifigkeiten der Platten, die in Tabelle 4 fett dargestellt sind. Bei der Bestimmung der Steifigkeiten dieser Platten strebte der Steifigkeitsparameter $C_{44}$ im Optimierungsprozess gegen unendlich. Daher wurde dieser geschätzt und anschließend näher untersucht (siehe Abschn. 5). Als Schätzwert wurde der Mittelwert der identischen Platten des jeweiligen Herstellers gewählt. Alle bestimmten Steifigkeiten $C_{i j}$ sowie die Rückrechnung in die Ingenieurkonstanten $\left(E_{i i}, G_{i j}\right)$ sind in Tabelle 4 angegeben.

\section{Optimierungsprozess}

Im Optimierungsprozess erwies es sich als sinnvoll, nicht alle Steifigkeitsparameter gleichzeitig zu bestimmen, sondern schrittweise vorzugehen. Vorerst wurden die Steifigkeitsparameter $C_{11}$ und $C_{22}$, welche bei der Sensitivitätsanalyse den größten Einfluss auf die Eigenfrequenzen aufweisen, einzeln bestimmt. Für die restlichen Steifigkeiten wurden die Schätzwerte $C_{i j}$ * verwendet. Sodann wurden die Steifigkeiten $C_{44}, C_{55}, C_{66}$ und schließlich alle zusammen angenähert. Die berechneten Startwerte $\left(C_{i j}^{*}\right)$ zeigten sich im Iterationsverfahren als genau genug, um in Richtung Zielparameter zu konvergieren. Es konnten für jede Platte mindestens 7 und maximal 14 korrespondierende Eigenformen (Modes) gefunden werden. Die höchste korrespondierende Eigenform wies eine Eigenfrequenz von $230 \mathrm{~Hz}$ (Mode 19) auf. Höhere Frequenzen konnten nicht mehr genau bestimmt werden. Der Vergleich zwischen den gemessenen und den (auf Basis der dynamisch bestimmten Steifigkeiten) berechneten Eigenfrequenzen zeigt, dass die maximale Abweichung bei 2,4\% liegt. Der Mittelwert aller Abweichungen beträgt 0,5\%. In Tabelle 6 sind am Beispiel von Platte A10 die theoretisch $\left(f_{\text {ber }}\right)$ und die 
Tabelle 5 Vergleich der gemessenen mit berechneten Durchbiegungen sowie Parameterstudie

Table 5 Comparison of the calculated and measured deformations and parameter study

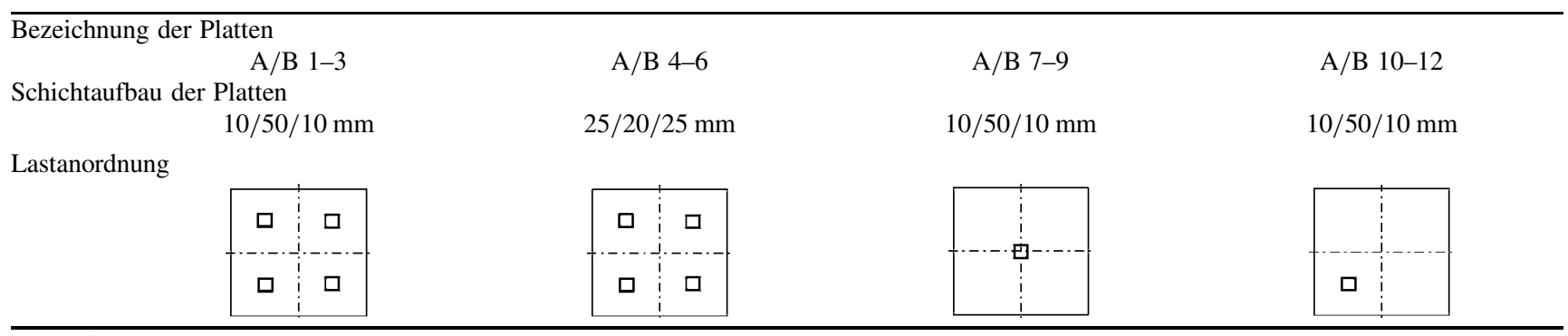

Durchbiegung gemessen/berechnet

Produkt A [mm]

\begin{tabular}{|c|c|c|c|c|c|c|c|c|c|c|c|c|c|c|c|}
\hline & $\mathrm{A} 1$ & & & & & $\Delta 6$ & & A8 & A9 & \multicolumn{2}{|c|}{ A10 } & \multicolumn{2}{|c|}{ A11 } & \multicolumn{2}{|c|}{ A12 } \\
\hline & - & & 33,3 & 6 & & & 1 & 19,9 & 20,5 & 3,7 & 8,4 & 9,2 & 8,5 &, 6 & 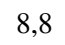 \\
\hline $2 / u$ &, 7 & 6 & 4 & 9 & 4 & 2 & 3 & 3 & 8 & ,3 & 7,4 &, 3 & 6 &, 1 & 8,1 \\
\hline$u_{3} / u_{3}^{*}$ & 3,6 & 7 & 3 & 6 & 8 & 5 & 712,3 & 812,4 & $13,112,7$ & 8,5 & 7,6 & 8,5 & 7,7 & 8,6 & 7,8 \\
\hline $4 / u_{4}^{*}$ & $20,919,4$ & $21,519,5$ & $21,0 \quad 19,9$ & $27,725,9$ & $23,323,3$ & $25,323,0$ & $9,0 \quad 8,4$ & $8,8 \quad 8,4$ & $9,3 \quad 8,7$ & 10,9 & 9,5 & 10,5 & 9,7 & 11,9 & 10,3 \\
\hline
\end{tabular}

Produkt B [mm]

\begin{tabular}{|c|c|c|c|c|c|c|c|c|c|c|c|c|}
\hline & B1 & B2 & B3 & B4 & B5 & B6 & B7 & B8 & B9 & B10 & B11 & B12 \\
\hline$u_{1} / u_{1}^{*}$ & $30,128,9$ & $30,629,2$ & $29,929,0$ & $27,027,1$ & $26,026,1$ & $26,326,3$ & $18,317,4$ & $18,3 \quad 17,0$ & $17,916,8$ & $7,8 \quad 7,4$ & $8,0 \quad 7,7$ & $7,8 \quad 7,3$ \\
\hline$u_{2} / u_{2}^{*}$ & $22,321,0$ & $22,721,3$ & $22,221,3$ & $19,218,9$ & $18,7 \quad 18,2$ & 18,4 & $11,310,8$ & $11,3 \quad 10,4$ & $11,1 \quad 10,4$ & $7,0 \quad 6,5$ & $7,2 \quad 6,8$ & $7,1 \quad 6,4$ \\
\hline$u_{3} / u_{3}^{*}$ & $22,621,1$ & $21,821,4$ & $22,321,1$ & $27,827,7$ & $27,927,4$ & $26,926,3$ & $11,410,7$ & $11,210,5$ & $11,1 \quad 10,4$ & $7,3 \quad 6,8$ & $7,5 \quad 7,2$ & $7,5 \quad 6,8$ \\
\hline$u_{4} / u_{4}^{*}$ & $18,7 \quad 17,2$ & $19,217,4$ & $18,8 \quad 17,4$ & $22,521,7$ & $22,421,6$ & $22,020,6$ & $7,9 \quad 7,4$ & $7,9 \quad 7,2$ & $7,7 \quad 7,1$ & $9,8 \quad 8,7$ & $9,9 \quad 9,1$ & $9,9 \quad 8,6$ \\
\hline
\end{tabular}

Parameterstudie der Durchbiegungen

Einfluss der Steifigkeitsänderung (10\%) auf die Durchbiegung
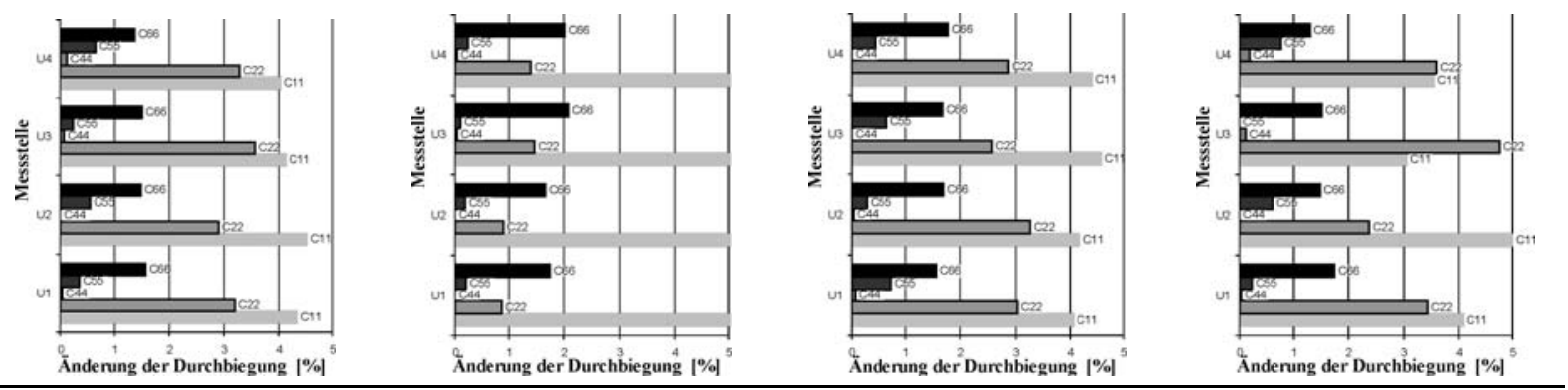

experimentell $\left(f_{\text {exp }}\right)$ bestimmten Eigenfrequenzen und Eigenformen (Modes) gegenübergestellt und die MAC-Werte aufgelistet.

\subsection{Vergleich der berechneten und der experimentell dynamisch bestimmten Steifigkeiten}

Indiesem Kapitel wird einerseits der Vergleich zwischen berechneten $\left(C_{i j}^{*}\right)$ und experimentell dynamisch ermittelten $\left(C_{i j}\right)$ Steifigkeiten dargestellt. Andererseits werden die ermittelten Steifigkeitswerte statistisch ausgewertet. Es wird überprüft, wie genau ingenieurmäßige Berechnungen sind und inwieweit sie auf der ,sicheren Seite“ liegen. Zu diesem Zweck wurden die berechneten Steifigkeiten $\left(C_{i j}^{*}\right)$ auf die Ingenieurmoduln $E_{i i}^{*}$ und $G_{i j}^{*}$ rückgerechnet, die dynamisch bestimmten Steifigkeiten $\left(C_{i j}\right)$ auf $E_{i i}$ und $G_{i j}$. In Abb. 5 sind die Steifigkeiten in 5 Diagrammen (Diagramm a bis Diagramm e) dargestellt. Die einzelnen Punkte entsprechen jeweils den dynamisch bestimmten Steifigkeiten $\left(E_{i i}\right.$ und $G_{i j}$ ), die horizontalen Geraden den berechneten Werten $\left(E_{i i}^{*}\right.$ und $G_{i j}^{*}$ ). Der grau hinterlegte Bereich markiert das Streuband zwischen Minimal- und Maximalwerten. Die grau gestrichelte Gerade in diesem Bereich entspricht dem Mittelwert aller experimentell dynamisch bestimmten Ingenieurmoduln.

Im Diagramm a und im Diagramm c der Abb. 5 werden die berechneten und die dynamisch gemessenen Werte der zwei E-Moduln verglichen. Der gemessene Elastizitätsmodul parallel zur Faserrichtung der Decklagen $\left(E_{11}\right)$ liegt bei den A-Platten und dünner Decklage unter den berechneten Werten. Die Platten des Produktes B mit dünner Decklage überschreiten den berechneten Wert hingegen deutlich. Bei dicken Decklagen $(25 \mathrm{~mm})$ überschreiten die Platten des Produktes A $E_{11}^{*}$ knapp, die B-Platten 
Tabelle 6 Vergleich der berechneten $\left(f_{\text {ber }}\right)$ und der experimentell bestimmten $\left(f_{\exp }\right)$ Eigenfrequenzen und Modes sowie zugehörige MAC-Werte (Modal Assurance Criterion)

Table 6 Comparison of calculated $\left(f_{\text {ber }}\right)$ and experimentally determined $\left(f_{\exp }\right)$ frequencies and mode-shapes together with corresponding MAC-values (Modal Assurance Criterion)

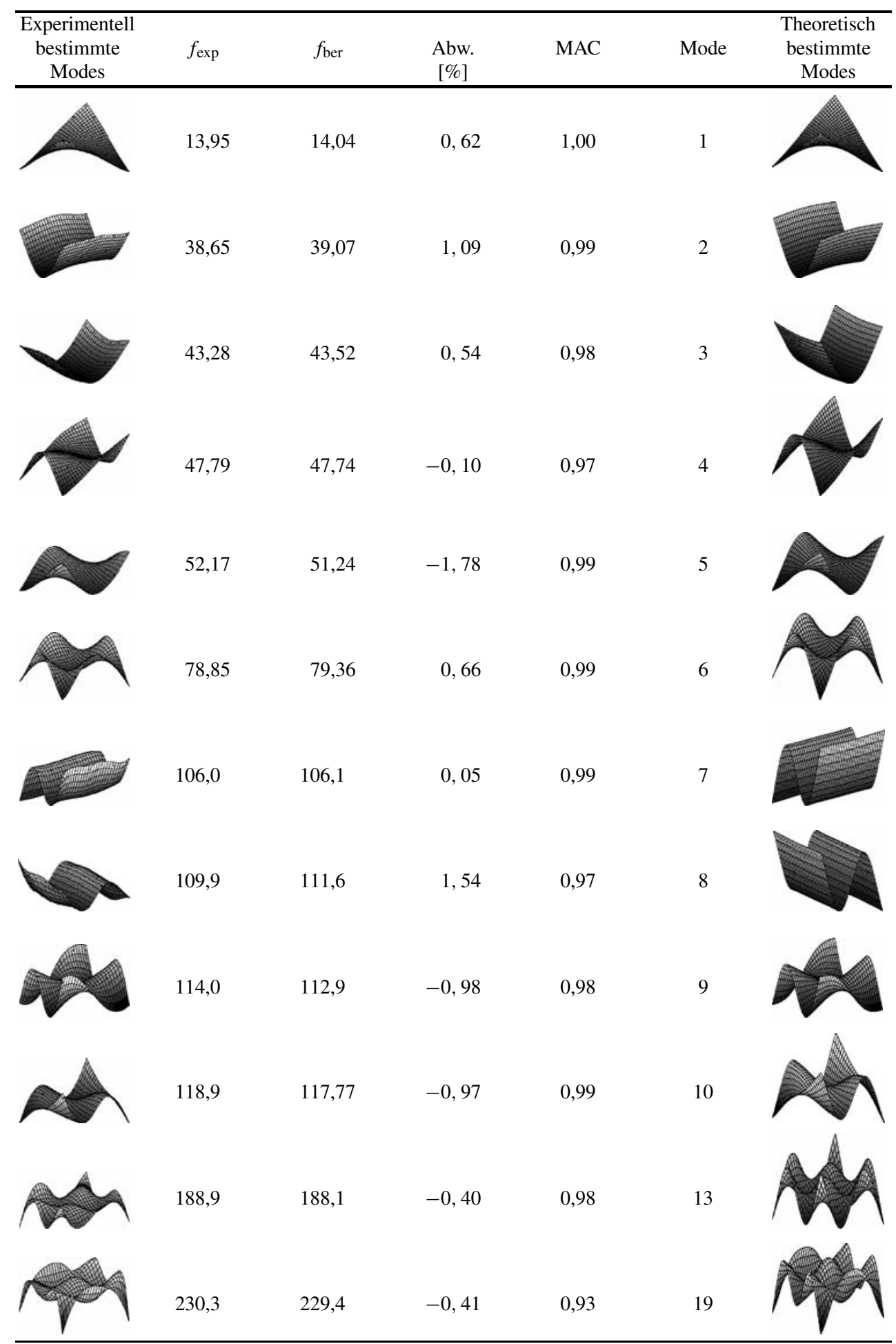

überschreiten die berechneten Werte wiederum deutlich. Der berechnete Elastizitätsmodul senkrecht zur Faserrichtung der Decklagen $\left(E_{22}^{*}\right)$ wird von allen Platten mit dicken Decklagen ( $25 \mathrm{~mm}$ ) knapp unterschritten. Auffällig sind die insgesamt größeren Streuwerte von Produkt A. Der dynamisch bestimmte Elastizitätsmodul von Platte A12 weicht beispielsweise um 17\% vom berechneten Elastizitätsmodul ab. Der Schubmodul $G_{23}$ variiert von Platte zu Platte extrem (Diagramm e). Plausible Werte liefern nur Platten des Produktes A mit dünner Decklage.

In Abb. 6 werden die dynamisch bestimmten Ingenieurskonstanten für die Platten mit den dünnen Decklagen 
Abb. 5 Vergleich experimentell dynamisch bestimmter $\left(G_{i j}, E_{i i}\right)$ und berechneter Steifigkeiten $\left(G_{i j}^{*}, E_{i i}^{*}\right)$

Fig. 5 Comparison of experimentally determined, dynamically elastic parameters $\left(G_{i j}, E_{i i}\right)$ and calculated elastic parameters $\left(G_{i j}^{*}, E_{i i}^{*}\right)$

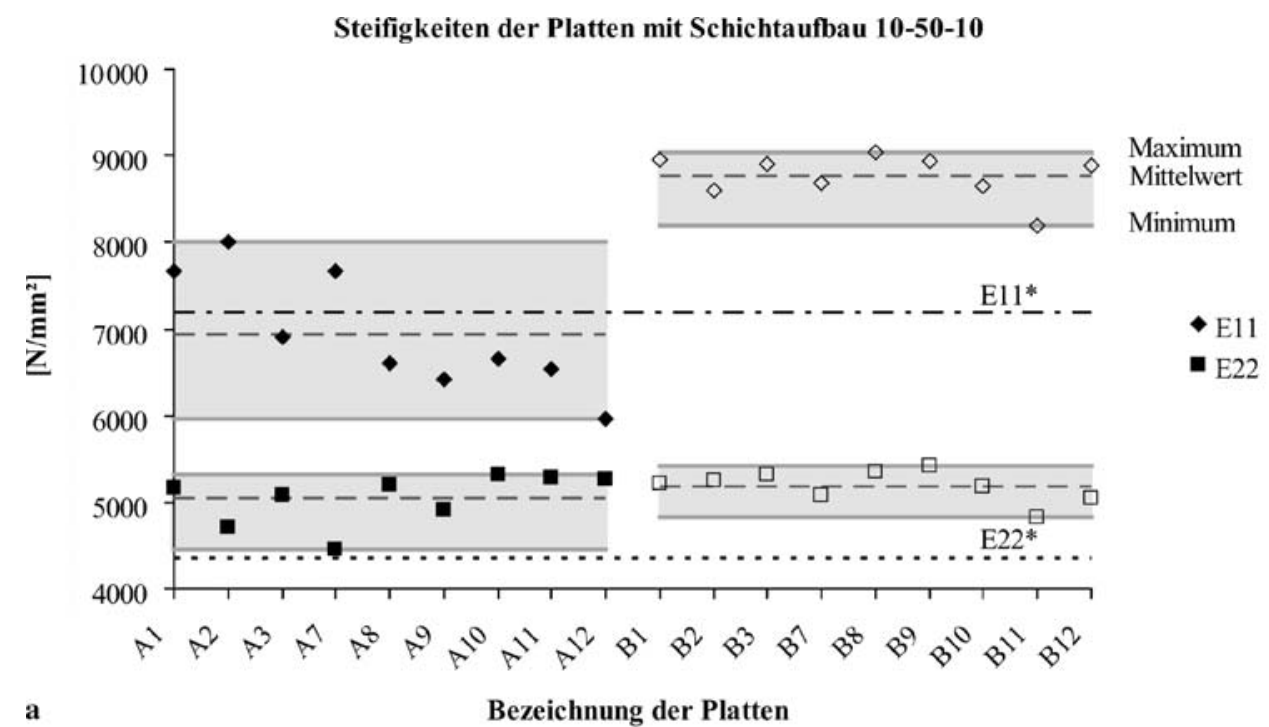

Steifigkeiten der Platten mit Schichtaufbau 10-50-10

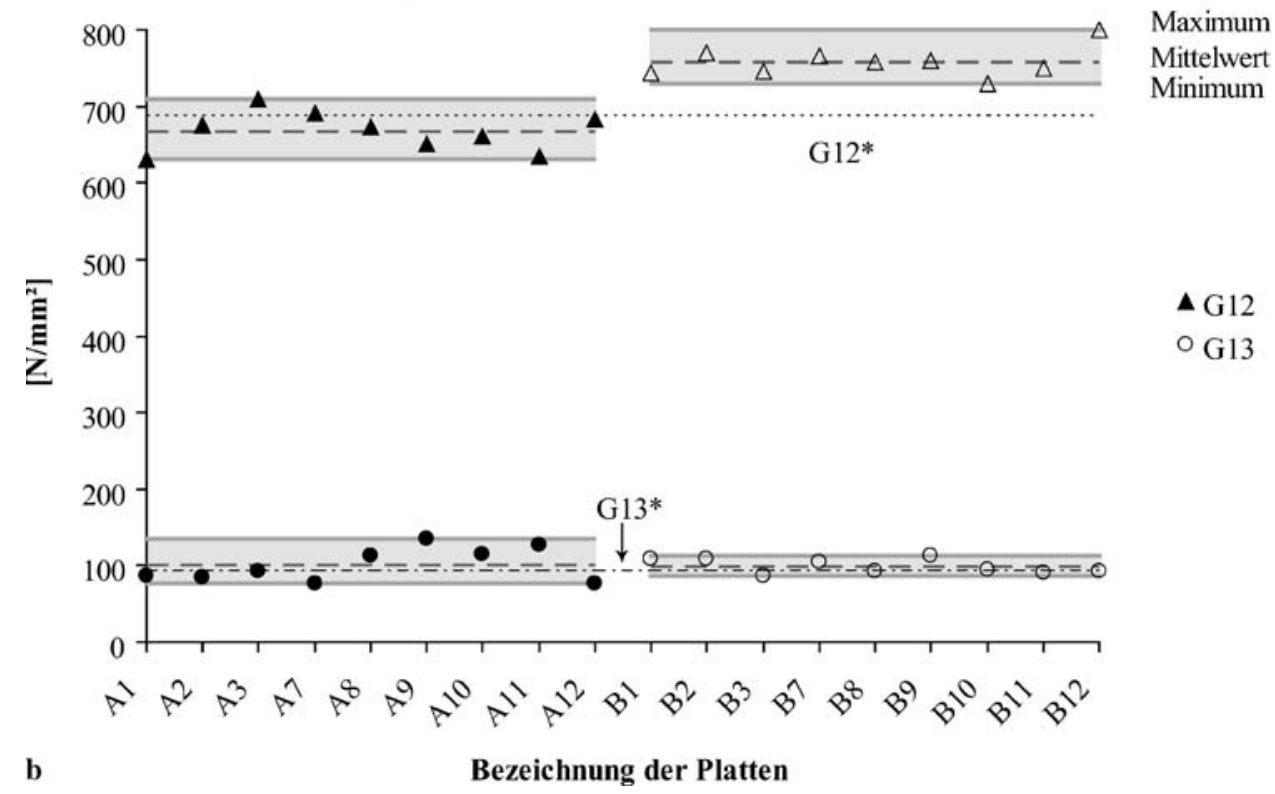

$(10 \mathrm{~mm})$ in einem normalverteilten Wahrscheinlichkeitsnetz dargestellt. Die Abszisse entspricht der Werteachse, die Ordinate beschreibt die Normalverteilung. Sämtliche Steifigkeiten sind normalverteilt, was durch die Linearität der Werte im Wahrscheinlichkeitsnetz bestätigt wird. Die Steigung der Geraden ist ein Maß für die Streuung. Der Schnittpunkt mit der Horizontalen bei $50 \%$ entspricht dem Mittelwert. Im Weiteren sind in Abb. 6 die Standardabweichungen sowie die Variationskoeffizienten angegeben. Eine statistische Auswertung ist nur bei den Platten mit dünnen Decklagen $(10 \mathrm{~mm})$ sinnvoll, da hier von jedem Produkt neun Platten untersucht werden konnten. Die Platten mit den breiten Decklagen $(25 \mathrm{~mm})$ werden statistisch nicht berücksichtigt, da die Stichprobenanzahl zu klein ist. Aus den größeren Steigungen der Regressionsgeraden von
Produkt B kann geschlossen werden, dass die Steifigkeiten dieses Produkts sehr viel weniger streuen, also einen durchgängig kleineren Variationskoeffizienten aufweisen, als die Platten des Produkts A.

\subsection{Verifizierung mit Biegeversuchen}

Die berechneten sowie gemessenen Durchbiegungen aller Platten an den vier Messstellen $U 1$ bis $U 4$ sind in Tabelle 5 aufgelistet. Der Mittelwert der prozentualen Abweichung der Berechnungen gegenüber den Messungen beträgt 5,9\%. Die maximale Abweichung wurde mit 14,9\% ermittelt (Messpunkt $U 4$ für Produkte A und B bei exzentrischer Belastung). Auffallend ist, dass die Abweichungen von den Messstellen und den Lasteinleitungsstellen abhängig sind. 
Abb. 5 fortgesetzt

Fig. 5 continued
Steifigkeiten der Platten mit Schichtaufbau 25/20/25

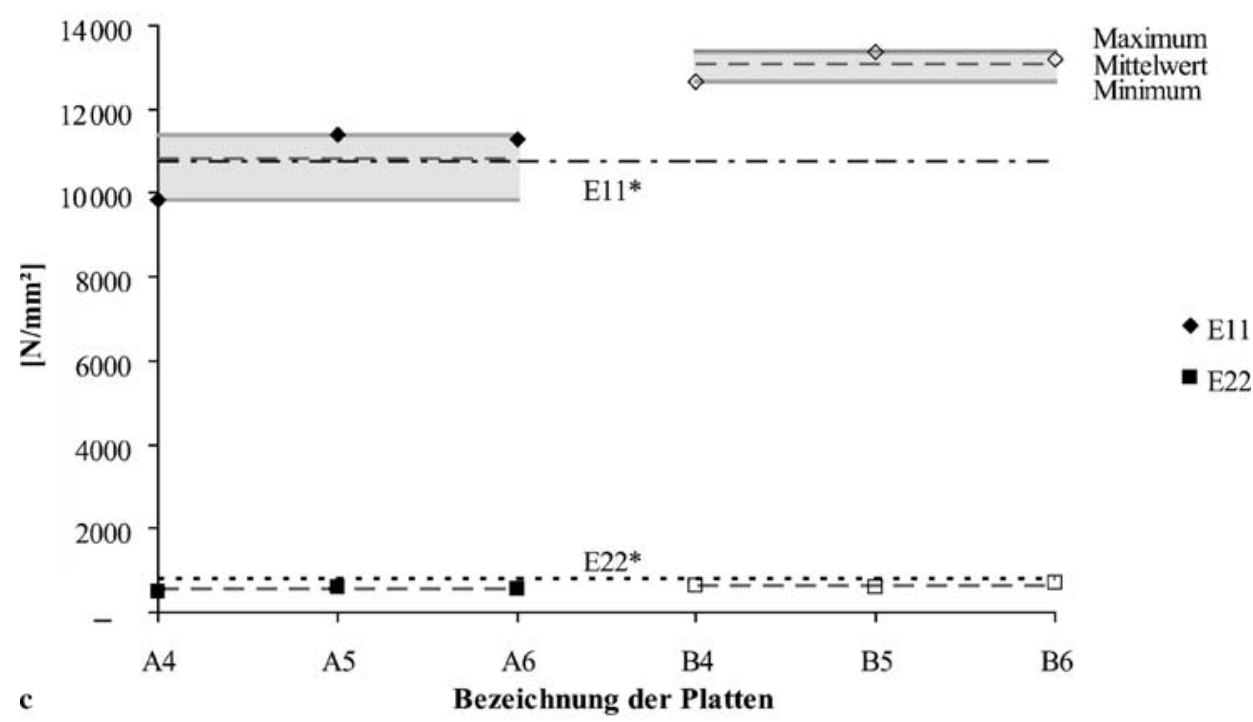

Steifigkeiten der Platten mit Schichtaufbau 25/20/25

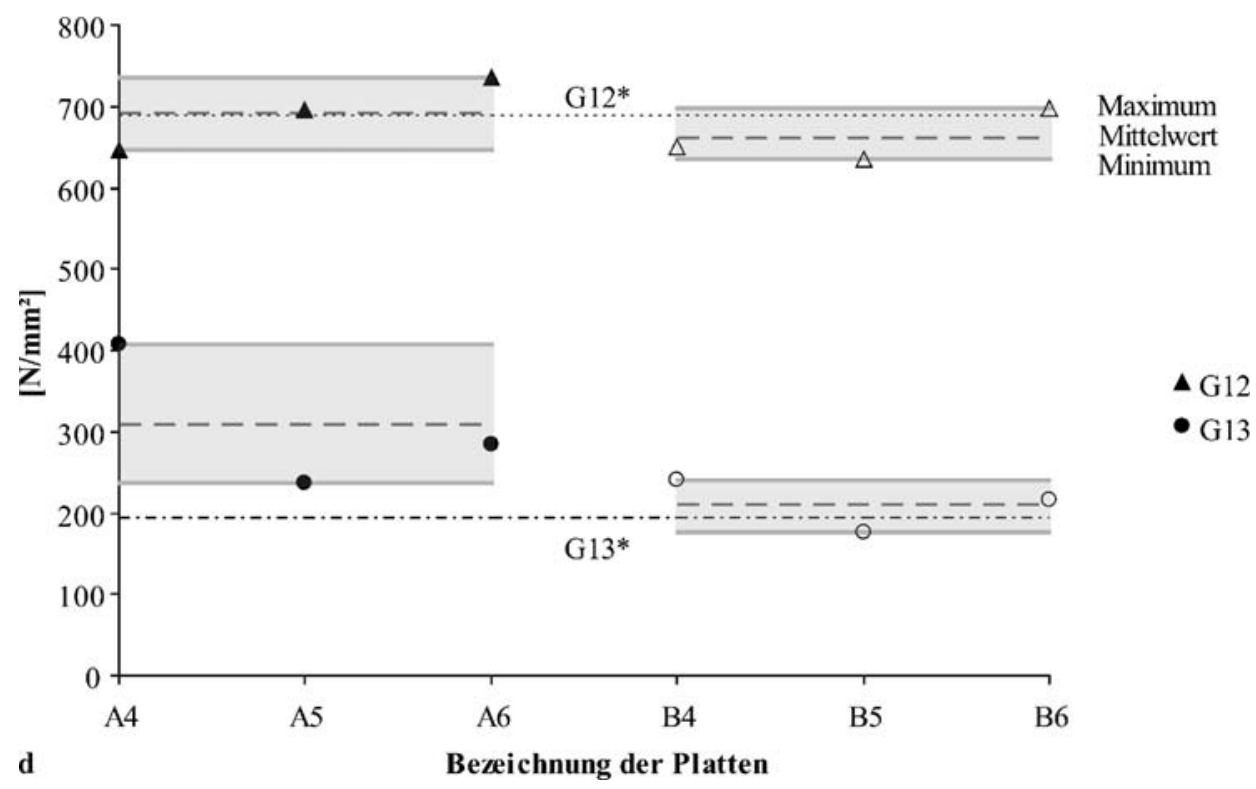

Die größte Diskrepanz zwischen berechneten und gemessenen Durchbiegungen zeigt sich bei einer exzentrischen Lasteinleitung und der Messstelle $U 4$, also nahe des Plattenrandes. Die gemessenen Durchbiegungen waren in 95\% aller Vergleiche grösser als die berechneten.

\section{Diskussion}

Die untersuchte Methode (Modalanalyse nach Gsell et al. (2007)) stellte sich als geeignet heraus, Steifigkeitsparameter von Brettsperrholzplatten zerstörungsfrei zu bestimmen. Die Eigenfrequenzen und Eigenformen konnten voll auto- matisch identifiziert und im Optimierungsprozess mit den im Modell berechneten Frequenzen abgeglichen werden. Die geringen Abweichungen zwischen theoretisch bestimmten und gemessenen Frequenzen zeigen, dass das verwendete Material- und Plattenmodell das mechanische Verhalten von Brettsperrholzplatten hinreichend genau beschreibt.

\subsection{Vergleich der berechneten} und gemessenen Durchbiegungen

Der Unterschied zwischen gemessenen und berechneten Durchbiegungen beträgt im Mittel ca. 6\%. Die gemessenen Durchbiegungen sind immer grösser als die berechneten. 
Abb. 5 fortgesetzt

Fig. 5 continued
Steifigkeiten der Platten mit Schichtaufbau 10-50-10

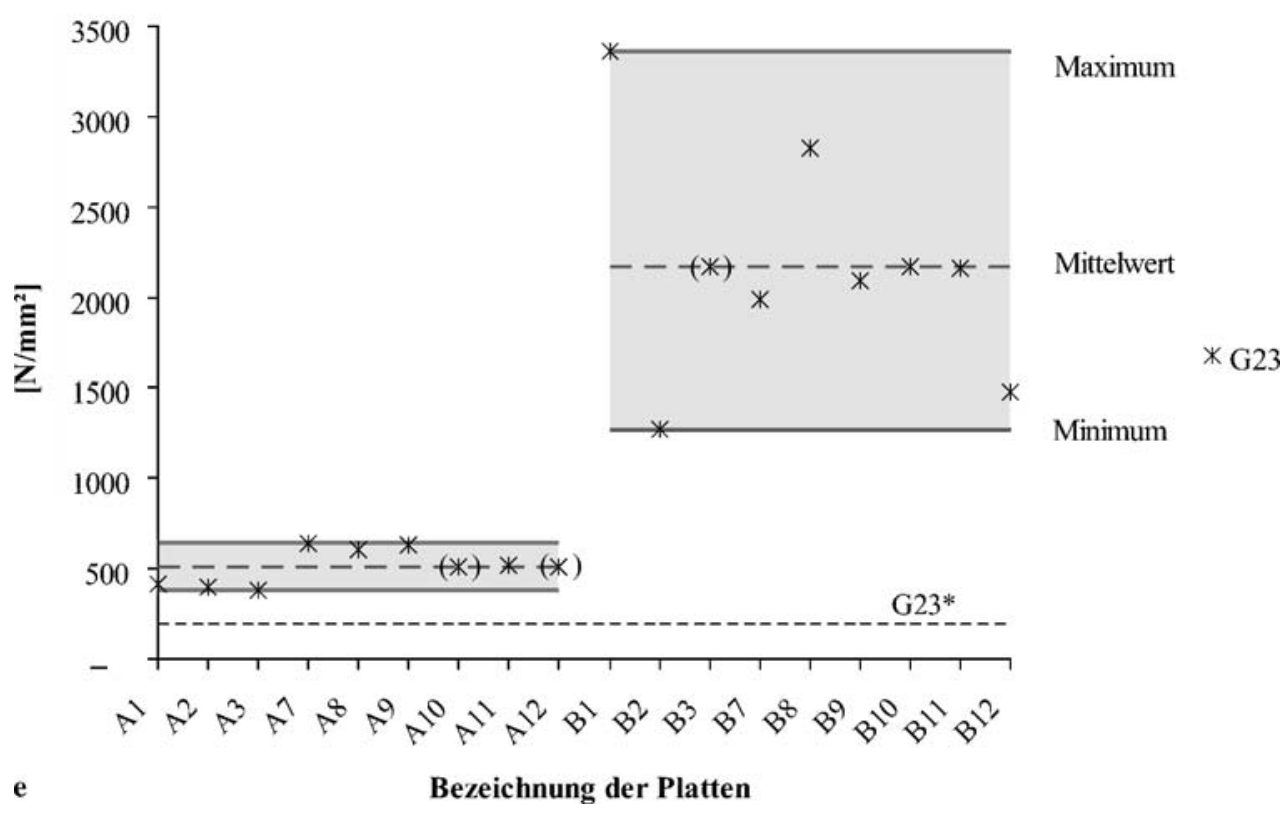

Da die Durchbiegungen linear von den elastischen Eigenschaften abhängen, bedeutet dies, dass auch die dynamisch bestimmten Parameter um 6\% zu hoch sind. Eine Erklärung für die Überschätzung der elastischen Parameter ist deren Abhängigkeit von der Belastungsgeschwindigkeit, welche bei den dynamischen Tests viel höher ist. Diese Erklärung deckt sich auch mit Aussagen von Görlacher (1984), in dessen Arbeit der dynamische Biegeelastizitätsmodul bei 95 Versuchskörpern im Mittel um 9\% über dem statischen lag.

Die Tatsache, dass sich die größte Diskrepanz zwischen berechneten und gemessenen Durchbiegungen bei exzentrischer Lasteinleitung und am Messpunkt $U 4$ zeigt, kann mit den Diagrammen in Tabelle 5 begründet werden. Im rechten Diagramm ist der Einfluss von $C_{44}$ auf die Durchbiegung an der Messstelle $U 4$ im Vergleich zu den anderen Belastungsarten am größten. Dies deutet wiederum darauf hin, dass die Steifigkeitsparameter $C_{44}$ für diese Platten zu ungenau bestimmt wurden.

\subsection{Vergleich der berechneten}

und der dynamisch bestimmten Steifigkeiten

Es können zusammenfassend zwei Aussagen zum Vergleich der berechneten und der dynamisch bestimmten Steifigkeiten gemacht werden:

1. Der Elastizitätsmodul senkrecht zur Faserrichtung $\left(E_{22}\right)$ der Decklagen wird in der Berechnung der Platten mit dem Schichtaufbau $25 \mathrm{~mm} / 20 \mathrm{~mm} / 25 \mathrm{~mm}$ überschätzt. Dieses Phänomen muss in der Annahme der Elasti- zitätsmoduln senkrecht zur Faser von Vollholz gesucht werden. Diese wurde basierend auf Literaturangaben (Bodig und Goodman 1973, Hearmon 1948, Neuhaus 1994) geschätzt. Es hat sich nun gezeigt, dass der Ansatz in der EN 338 (2003) zur Berechnung des Elastizitätsmoduls $E_{22}$ von Vollholz aus dem Elastizitätsmodul parallel zur Faser, d.h. $E_{22}=E_{11} / 30$ zu einer besseren Übereinstimmung zwischen Versuch und Berechnung geführt hätte. Eine Berechnung mit dem Ansatz aus EN 338 (2003) ergibt für die betreffenden Platten einen Wert von $615 \mathrm{~N} / \mathrm{mm}^{2}$. Nur noch die Steifigkeiten der Platten des Produktes A sind dann geringfügig überschätzt.

2. Die experimentell dynamisch bestimmten Steifigkeiten $C_{44}$ bzw. $G_{23}$ weisen einen extrem hohen Variationskoeffizienten auf. Sie überschätzen den berechneten Wert $G_{23}^{*}$ außerdem enorm. Die Bestimmung von $C_{44}$ muss daher näher untersucht werden.

\subsection{Steifigkeitsparameter $C_{44}$ (Schubmodul $G_{23}$ )}

Problem

Die Bestimmung von plausiblen Werten für $C_{44}$ war nur bei Platten des Produktes A mit den dünnen Decklagen möglich. Dass dieser Parameter schwer zu bestimmen und die Genauigkeit dieses Parameters sehr gering ist, zeigten die Sensitivitätsanalysen. Hier stiegen bei einer Änderung von $C_{44}$ um $10 \%$ die Frequenz-Änderungen erst ab Mode 14 über 0,4\%, was als Mindestwert angenommen wird, um einen Steifigkeitsparameter bestimmen zu können. Die 
Abb. 6 Statistische

Auswertung der

Steifigkeitsmoduln $\left(G_{i j}, E_{i i}\right)$

der untersuchten Platten

Fig. 6 Statistic evaluation of

the elastic parameters $\left(G_{i j}\right.$,

$E_{i i}$ ) of the examined panels
E-Modul E11 und E22 im normalverteilten Wahrscheinlichkeitsnetz

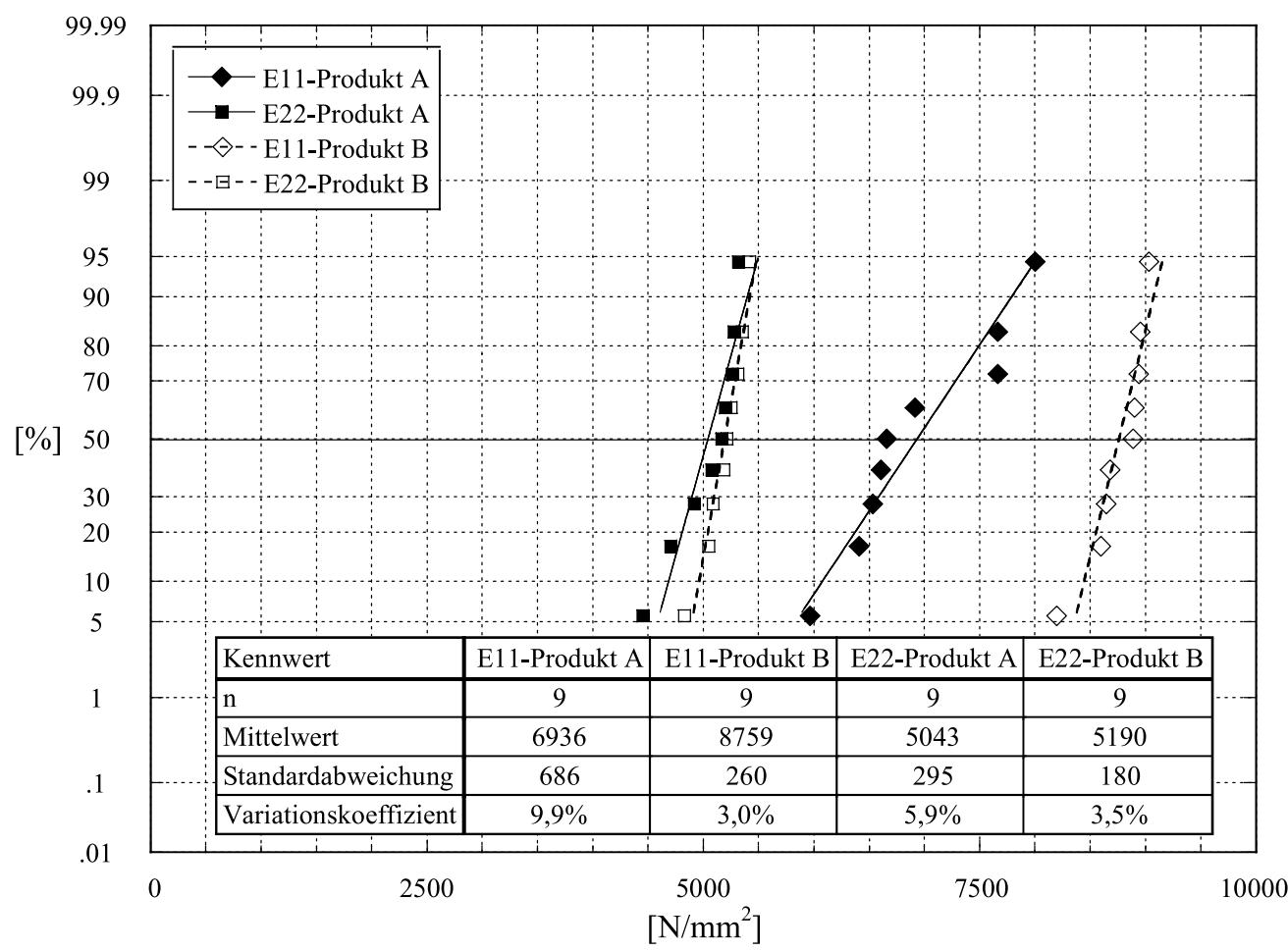

G-Modul G12 und G13 im normalverteilten Wahrscheinlichkeitspapier

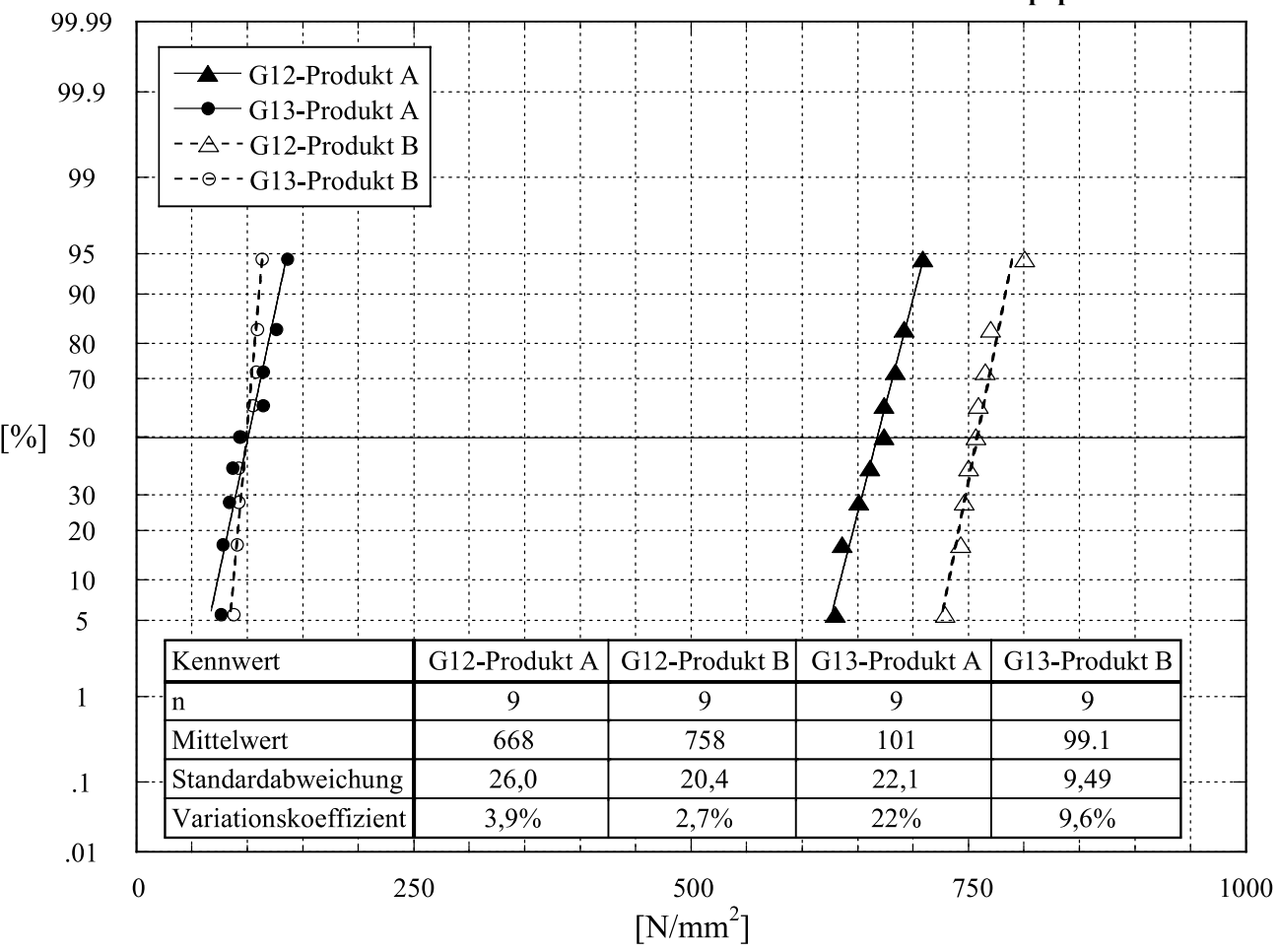

Frequenzen in dieser Größenordnung konnten jedoch experimentell größtenteils nicht bestimmt werden. Auch die Verifizierungen durch Biegeversuche sind für $C_{44}$ nicht aussagekräftig, wie die Sensitivitätsanalyse gezeigt hat. Andere Steifigkeitsparameter weisen einen 25-mal höheren Einfluss auf die Durchbiegung auf. Eine Ausnahme bildet die exzentrische Lasteinleitung. Der Vergleich der gemessenen $\left(u_{4}\right)$ und der berechneten Durchbiegungen $\left(u_{4}^{*}\right)$ gibt zwar keine Auskunft über die Qualität des dynamisch bestimmten $C_{44}$, bestätigt aber, dass dieses $C_{44}$ sehr ungenau ist. 


\section{Bedeutung des Parameters $C_{44}$}

$C_{44}$ ist in der Ingenieursprache als $G_{23}$ bekannt und setzt sich aus dem so genannten Rollschubmodul der Decklagen und dem Schubmodul der Mittellage zusammen (Tabelle 3). Ob der Parameter $G_{23}$ für statische Berechnungen relevant ist, hängt vom statischen System und der Geometrie der Platte ab. Bei statischen Berechnungen von Balken und Platten ist bekannt, dass der Einfluss der Schubsteifigkeit auf die Durchbiegung zunimmt, je kürzer die Spannweite des Trägers im Verhältnis zu seiner Dicke ist und je kleiner seine Schubsteifigkeit im Verhältnis zur Biegesteifigkeit ist (Ehlbeck 1967).

Sowohl Schwingungsberechnungen als auch statische Berechnungen können auf der Theorie basieren, dass die Summe aller Energieänderungen Null ist (Prinzip vom Minimum des elastischen Gesamtpotentials). In beiden Berechnungen hängt das so genannte ,elastische Potential“" direkt von der Steifigkeitsmatrix $C_{i j}$ und somit auch von dem einzelnen Parameter $C_{44}$ ab. Daher kann angenommen werden, dass der Einfluss von $C_{44}$ auf die Eigenfrequenzen tendenziell zunimmt, wenn auch der Einfluss dieses Parameters in statischen Berechnungen seitlich aufgelagerter Platten größer wird. Um zu überprüfen, welche Geometrien und Eigenschaften dreischichtige, symmetrische Brettsperrholzplatten aufweisen müssen, damit der elastische Parameter $C_{44}$ experimentell dynamisch bestimmt werden kann und welchen Einfluss dieser Parameter dann auf statische Berechnungen hat, wurde eine Parameterstudie durchgeführt.

\section{Parameterstudie}

In der Parameterstudie wurden die Frequenzen zweier Platten mit den in Tabelle 1 geschätzten $C_{i j}^{*}$-Werten berechnet. Daraufhin wurde $C_{44}^{*}$ um jeweils $10 \%$ erhöht und die Veränderung der Eigenfrequenzen in die Diagramme in Abb. 7 übertragen. Dieser Vorgang wurde sukzessive auf verschiedene geometrische und physikalische Verhältnisse ausgeweitet. Zuerst wurden Analysen in Abhängigkeit der Plattendicke zur Seitenlänge $b$ (Seite senkrecht zur Faserrichtung der Decklagen) durchgeführt $(h / b)$. Hierbei wurde die Plattendicke schrittweise erhöht. Daraufhin wurde der Einfluss des Parameters $C_{44}$ in Abhängigkeit der Seitenverhältnisse $a / b$ und zuletzt in Abhängigkeit des Verhältnisses $G_{23} / E_{22}$ untersucht. Die Sensitivitätsanalysen wurden nur bis zu Mode 15 durchgeführt, da in der experimentellen Modalanalyse meistens keine höheren Eigenfrequenzen gemessen werden konnten. Die schwarzen fettgedruckten Linien geben die maximale Sensitivität für die verschiedenen Parameterverhältnisse an, die schwarzen Quadrate die maximale Sensitivität der untersuchten Platten. Die Sprünge in den einzelnen Kurven der unter- schiedlichen Modes sind mit der Tatsache zu erklären, dass sich mit veränderten Seiten- und Steifigkeitsverhältnissen auch die Reihenfolge der einzelnen Schwingungsformen ändern.

\section{Einfluss der Geometrie auf die Bestimmbarkeit von $G_{23}$}

Die Auswertungen zeigen, dass mit kürzer werdender Seitenlänge $a$ (Seite parallel zur Faserrichtung der Decklagen), mit abnehmendem Verhältnis $G_{23}^{*} / E_{22}^{*}$ und mit zunehmender Plattendicke $h$ die Sensitivität steigt.

Berechnet man das Verhältnis $G_{23}^{*} / E_{22}^{*}$ mit den in Gl. 3 bis 6 angegebenen Verhältnissen, erhält man folgende Gl. 9:

$\frac{G_{23}^{*}}{E_{22}^{*}}=\frac{5 h^{4}}{\left(40 h-36 a_{2}\right) \cdot\left(h^{3}+19 a_{2}^{3}\right)}$.

Eine mathematische Auswertung dieser Gleichung ergibt, dass das Verhältnis $G_{23}^{*} / E_{22}^{*}$ für zunehmende $a_{2}$, also für größer werdende Mittelschichten, in den Bereichen zwischen ca. $a_{2}=1 / 7 \cdot h$ und $a_{2}=4 / 5 \cdot h$ streng monoton fallend ist. $h$ beschreibt hier die Dicke der Platten. Das Verhältnis $G_{23}^{*} / E_{22}^{*}$ nimmt also ab und die Sensitivität zur Bestimmung von $C_{44}$ wird größer. Das bestätigt die Beobachtung, dass für die Platten mit dünnen Decklagen die Steifigkeiten $C_{44}$ besser bestimmt werden konnten.

Je dünner die Decklagen von 3-schichtigen, symmetrischen Brettsperrholzplatten sind, je dicker die gesamte Platte und je kleiner die Seite $a$ (parallel zur Faserrichtung der Decklagen), desto besser kann der Steifigkeitsparameter $C_{44}\left(G_{23}\right)$ bestimmt werden. In einer weiteren, stichprobenartigen (statischen) Parameterstudie wurde der Einfluss des Steifigkeitsparameter $C_{44}$ auf die Durchbiegung allseitig gelagerter Platten unter zentrischer Lasteinleitung untersucht. Der Steifigkeitsparameter $C_{44}$ wurde jeweils um $10 \%$ erhöht und die Änderung der Durchbiegungen der Platte unter gleichen Verhältnissen wie in der dynamischen Parameterstudie berechnet. Zusammenfassend kann betreffend der statischen Parameterstudie festgehalten werden, dass mit dickerer Decklage, mit dünnerer Gesamtdicke der Platte und mit steigender Seitenlänge $a$ der Einfluss von $C_{44}\left(G_{23}\right)$ auf die Durchbiegung sinkt.

\section{Schlussfolgerungen}

Es wurde eine Methode zur zerstörungsfreien Bestimmung elastischer Eigenschaften von dreischichtigen quadratischen Brettsperrholzplatten mit bezüglich der Mittellage symmetrischem Aufbau untersucht und folgende Ergebnisse gefunden:

- Alle für statische Berechnungen relevanten Steifigkeitsparameter sind direkt am fertigen Produkt bestimmbar. 


\section{Sensitivität der Steifigkeit $C_{44} *$}

10/50/10 mm
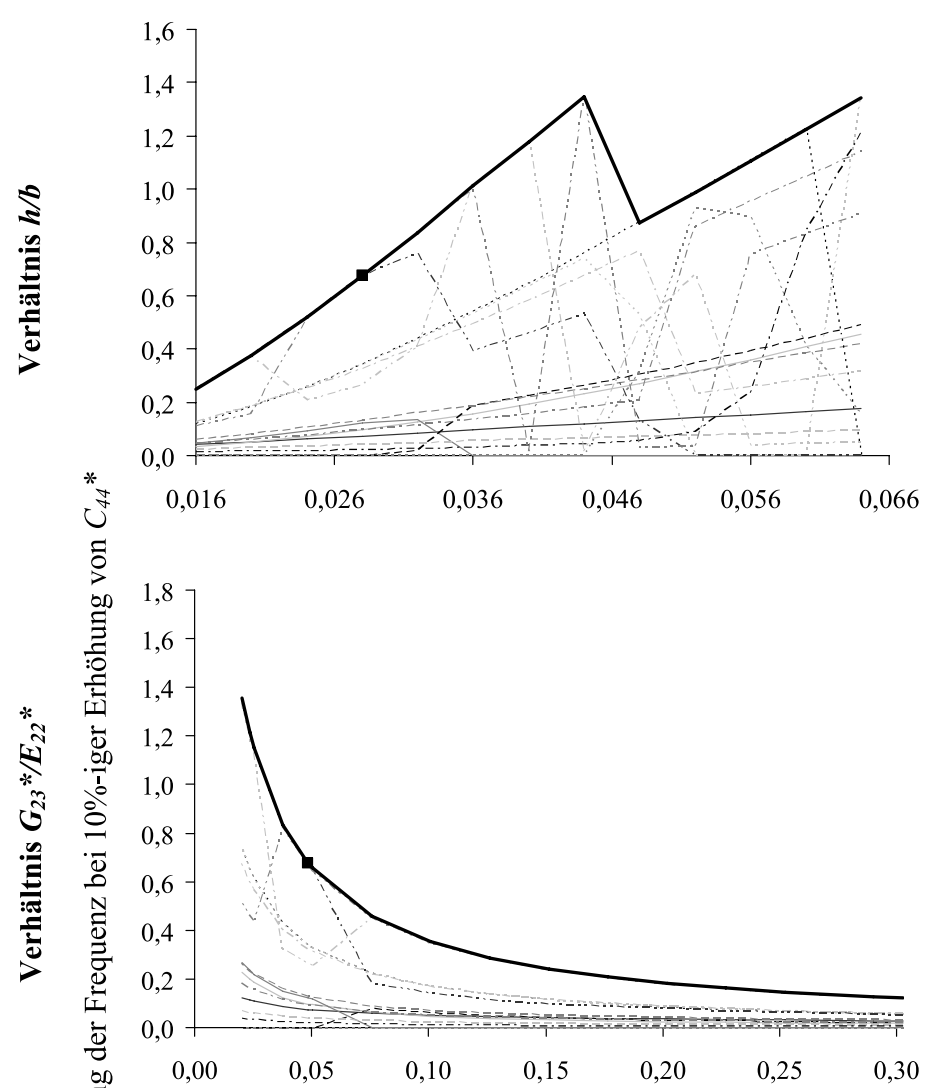

25/20/25 mm
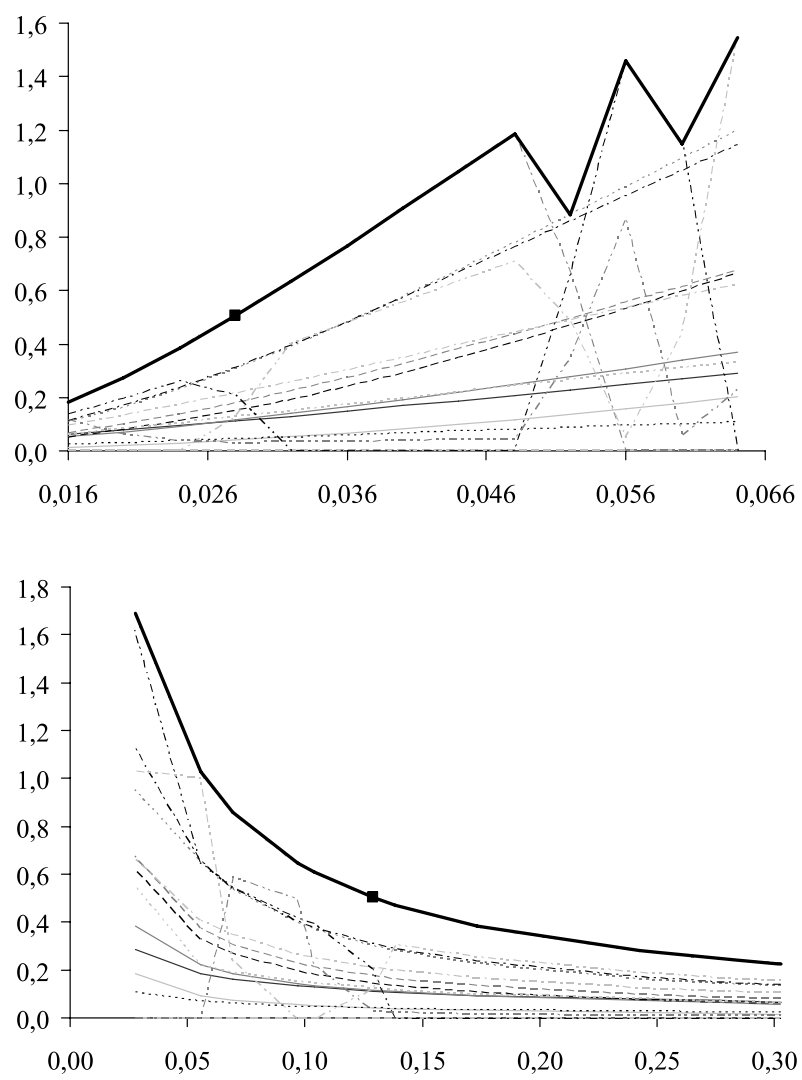
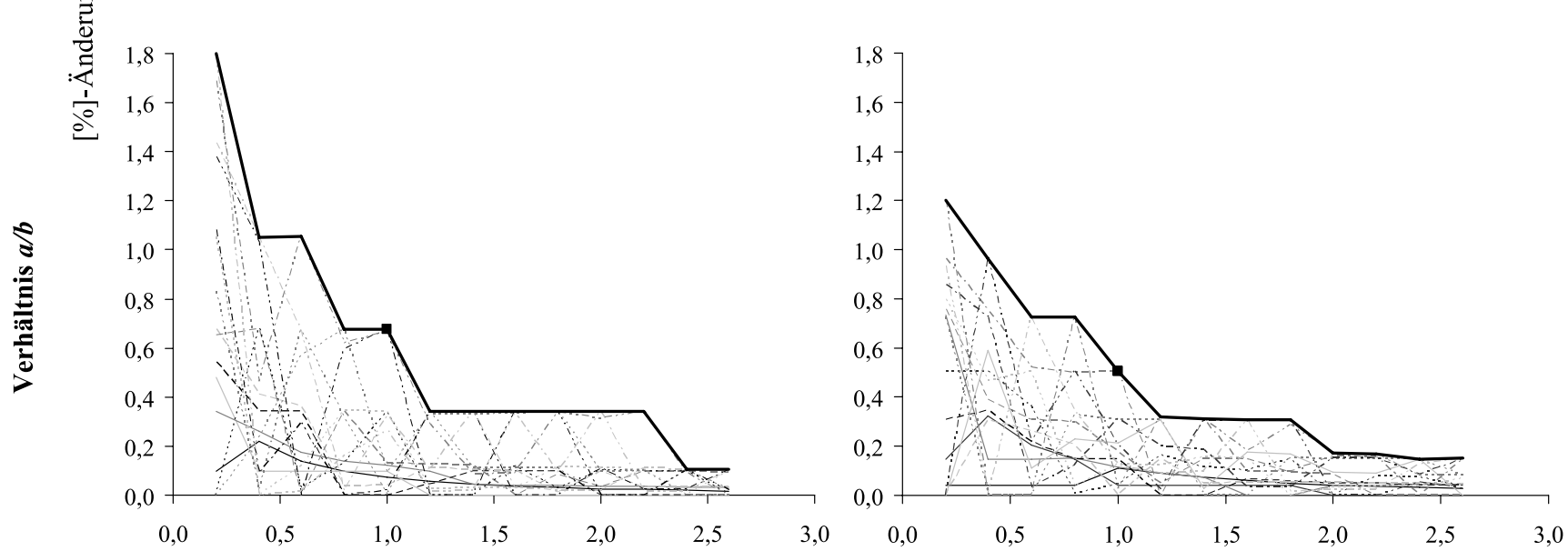

Abb. 7 Sensitivitätsanalyse von $C_{44}^{*}$ in Abhängigkeit von $G_{23}^{*} / E_{22}^{*}$, den Seitenlängen $a / b$ und dem Verhältnis Plattendicke zur Seitenlänge $h / b$. Die unterschiedlich gekennzeichneten Linien stellen die verschiedenen Modes dar

Fig. 7 Sensitivity analysis of $C_{44}^{*}$ as a function of $G_{23}^{*} / E_{22}^{*}$, the side-lengths $a / b$ and the ratio of panel thickness and side-length $h / b$. The differently marked lines describe the different modes

Durch einen Vergleich von Durchbiegungen aus Biegeversuchen und den Durchbiegungen aus einem Simulationsmodell unter Einsetzen der experimentell dynamisch bestimmten elastischen Parameter wurde die Richtigkeit dieser Steifigkeitsparameter verifiziert.
- Die untersuchte Methode weist für den Ingenieurbau eine ausreichende Genauigkeit auf.

- Bei ingenieurmäßiger Berechnung mit der Verbundtheorie und unter der Annahme, es handle sich um normales Bauholz der Festigkeitsklasse C24, können Steifigkei- 
ten überschätzt werden, vor allem, wenn die Mittellagen seitlich nicht miteinander verklebt sind. Unsichere, aber auch unwirtschaftliche Ergebnisse können sich ergeben, insbesondere dann, wenn keine ausreichende Kenntnis der tatsächlich vorhandenen mechanischen Eigenschaften vorhanden ist.

- Verschiedene Produktionsverfahren und seitliche Verklebungen der Mittellamellen haben einen großen Einfluss auf die Streuung der elastischen Eigenschaften von Brettsperrholzplatten.

- Die Steifigkeit $C_{44}\left(G_{23}\right)$ konnte nicht für alle Platten bestimmt werden. Eine statistische Auswertung dieser experimentell dynamisch bestimmten Steifigkeiten $C_{44}$ zeigte große Streuungen. Die ingenieurmäßig berechneten $C_{44}$-Werte (Bodig und Jayne 1993) lagen weit unter den dynamisch bestimmten. Eine Verifizierung von $C_{44}$ ist durch Biegeversuche nicht möglich. Die Bestimmbarkeit des Steifigkeitsparameters $C_{44}$ hängt vom Plattenaufbau und von den Seitenverhältnissen ab. Je größer die Plattendicke, je dünner die Decklagen und je kleiner die Seite a (Seite parallel zur Faserrichtung der Decklagen) von 3-schichtigen, symmetrischen Brettsperrholzplatten sind, desto besser kann der Steifigkeitsparameter $C_{44}\left(G_{23}\right)$ bestimmt werden.

- Der Parameter $C_{55}\left(G_{13}\right)$ hat größeren Einfluss auf die Frequenzen als $C_{44}\left(G_{23}\right)$, da die Sensitivität der Steifigkeiten steigt, wenn das Verhältnis $G_{13} / E_{11}$ bzw. $G_{23} / E_{22}$ kleiner wird. Das Verhältnis $G_{13} / E_{11}$ der in diesem Forschungsprojekt behandelten Platten ist um den Faktor 4 bis 10 kleiner als $G_{23} / E_{22}$. Daher ist $G_{13}$ bei diesen Platten besser bestimmbar als $G_{23}$.

\section{Ausblick}

Ob das vorgestellte Verfahren zur zerstörungsfreien Bestimmung der Steifigkeiten von Brettsperrholzplatten auch für andere Schichtaufbauten und Plattenabmessungen, wie sie z.B. im Brückenbau verwendet werden, seine Gültigkeit hat, werden derzeit laufende Folgeprojekte an der Empa zeigen. In diesen Forschungsarbeiten werden Brettsperrholzplatten verschiedener Dicken und Dimensionen, aber auch mit mehr als drei Lagen untersucht.

Danksagung Die finanzielle Unterstützung durch den Fonds zur Förderung der Wald- und Holzforschung im Schweizerischen Bundesamt für Umwelt (BAFU) wird verdankt. Ebenfalls Dank gilt den Plattenproduzenten Schilliger Holz AG, Küssnacht am Rigi $(\mathrm{CH})$, und Pius Schuler AG, Rothenthurm $(\mathrm{CH})$, welche das Versuchsmaterial zur Verfügung stellten.

\section{Literatur}

Albers K (1970) Querdehnungs- und Gleitzahlen, sowie Schub- und Scherfestigkeiten von Holzwerkstoffen. Dissertation, Universität Hamburg

Altenbach H, Altenbach J, Rikards R (1996) Einführung in die Mechanik der Laminat- und Sandwichtragwerke: Modellierung und Berechnung von Balken und Platten aus Verbundwerkstoffen. Deutscher Verlag für Grundstoffindustrie Stuttgart, Stuttgart

Blaß HJ, Görlacher R (2003) Bemessung im Holzbau: Brettsperrholz - Berechnungsgrundlagen. Holzbau-Kalender. Bruderverlag, Karlsruhe, pp 580-598

Bodig J, Goodman JR (1973) Prediction of Elastic Parameters for Wood. Wood Sci 5(4):249-264

Bodig J, Jayne BA (1993) Mechanics of wood and wood composites. Krieger Publishing Company, Malabar, Florida

Boström L, Holmqvist C (1999) State-of-the-art on machine strength grading. In: Proceedings of the 1st International RILEM Symposium on Timber Engineering, Stockholm, Sweden, pp 513-522

CEN (1995) EN 789: Holzbauwerke - Prüfverfahren - Bestimmung der mechanischen Eigenschaften von Holzwerkstoffen

CEN (2003) EN 338: Bauholz für tragende Zwecke - Festigkeitsklassen

CEN (2003) EN 408: Holzbauwerke - Bauholz für tragende Zwecke und Brettschichtholz - Bestimmung einiger physikalischer und mechanischer Eigenschaften

Czaderski C, Steiger R, Howald M, Olia S, Gülzow A, Niemz P (2007) Versuche und Berechnungen an allseitig gelagerten 3schichtigen Brettsperrholzplatten. Holz Roh- Werkst 65(5): 383-402

DIN Deutsches Institut für Normung (Berlin) (1979) DIN 68364: Kennwerte von Holzarten - Festigkeit, Elastizität, Resistenz

DIN Deutsches Institut für Normung (Berlin) (2004) DIN 1052: Entwurf, Berechnung und Bemessung von Holzbauwerken Allgemeine Bemessungsregeln und Bemessungsregeln für den Hochbau. Beuth, Berlin

Ehlbeck J (1967) Durchbiegungen und Spannungen von Biegeträgern aus Holz unter Berücksichtigung der Schubverformung. Dissertation, Karlsruhe, p 136

Görlacher R (1984) Ein neues Messverfahren zur Bestimmung des Elastizitätsmoduls von Holz. Holz Roh- Werkst 42:219-222

Görlacher R (2002) Ein Verfahren zur Ermittlung des Rollschubmoduls von Holz. Holz Roh- Werkst 60:317-322

Gsell D, Feltrin G, Schubert S, Steiger R, Motavalli M (2007) Cross laminated timber plates: Evaluation and verification of homogenized elastic properties. J Struct Eng 133(1):132-138

Hearmon RFS (1948) Elasticity of wood and plywood. Department of Scientific and Industrial Research, London

Maia NMM (1997) Theoretical and experimental modal analysis. Research Studies Press etc., Taunton, Somerset, England

Neuhaus H (1994) Lehrbuch des Ingenieurholzbaus. B.G. Teubner, Stuttgart

Reddy JN (1984) A Simple Higher-Order Theory for Laminated Composite Plates. J Appl Mech-Trans ASME 51(4):745-752

Sinclair AN, Farshad M (1987) A comparison of three methods for determining elastic constants of wood. J Test Eval 15(2):77-86

Stamer J (1935) Elastizitätsuntersuchungen an Hölzern. Archive of Applied Mechanics (Ingenieur Archiv). Springer, Berlin, Heidelberg

Steiger R (1997) Sortierung von Rund- und Schnittholz mittels Ultraschall. Holzforsch Holzverwert 49(2):28-35 